\title{
Unveiling the nucleation and coarsening mechanisms of solution-derived self-assembled epitaxial $\mathrm{Ce}_{0.9} \mathrm{Gdd}_{0.1} \mathrm{O}_{2-\mathrm{y}}$
}

\section{nanostructures}

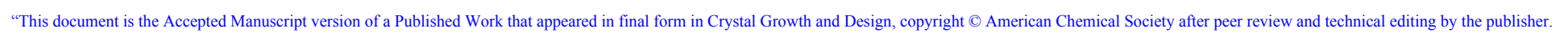
To access the final edited and published work see: https://dx.doi.org/10.1021/acs.cgd.6b01358"

Albert Queraltó ${ }^{* 1}$, María de la Mata $^{1,2}$, Jordi Arbiol ${ }^{2,3}$, Ruben Hühne $^{4}$, Xavier Obradors ${ }^{1}$, Teresa Puig ${ }^{1}$

${ }^{1}$ Institut de Ciència de Materials de Barcelona, Consejo Superior de Investigaciones Científicas (ICMABCSIC), Campus UAB, 08193 Bellaterra, Catalonia, Spain.

${ }^{2}$ Catalan Institute of Nanoscience and Nanotechnology (ICN2), CSIC and The Barcelona Institute of Science and Technology (BIST), Campus UAB, Bellaterra, 08193 Barcelona, Catalonia, Spain

${ }^{3}$ ICREA, Passeig Lluís Companys 23, 08010 Barcelona, Catalonia, Spain

${ }^{4}$ Institute for Metallic Materials, IFW Dresden, P.O. Box 270116, 01171 Dresden, Germany.

KEYWORDS: epitaxial nanostructures, $\mathrm{Ce}_{1-\mathrm{x}} \mathrm{Gd}_{\mathrm{x}} \mathrm{O}_{2-\mathrm{y}}$, Chemical Solution Deposition, nucleation, coarsening, Rapid Thermal Annealing

\begin{abstract}
Self-assembling approaches based on chemical solution deposition (CSD) are ideal methods for the costeffective production of epitaxial nanostructures with high-throughput. Therefore, an in-depth investigation of the nucleation and coarsening processes involved in the self-assembling of nanostructures is mandatory to achieve a good control over nanostructure shape, dimensions and orientation. Heteroepitaxial $\mathrm{Ce}_{0.9} \mathrm{Gd}_{0.1} \mathrm{O}_{2-\mathrm{y}}(\mathrm{CGO})$ is an ideal model system to unveil the underlying nanostructure development mechanisms in addition to their promising properties for catalysis, gas sensors and ionic
\end{abstract}


conductivity. Rapid thermal annealing furnaces have been used to study separately the thermodynamic and kinetic nucleation and coarsening mechanisms of self-assembled CGO isotropic and anisotropic nanostructures based on strain-engineering and surface energies control. Different CGO nanoislands are obtained: isotropic (001)CGO nanodots are grown on (001)-oriented $\mathrm{Y}_{2} \mathrm{O}_{3}: \mathrm{ZrO}_{2}$ (YSZ) and $\mathrm{LaAlO}_{3}$ (LAO) substrates, whereas (011)LAO substrates promote the growth of elongated (011)CGO nanowires. HRTEM and RHEED analyses are used to study the early stages of nucleation, as well as the shape and interfacial structure of CGO nanostructures. A systematic study with the heating ramp, annealing temperature and time, and strain in combination with thermally-activated theoretical models provides information on the nucleation behavior, nucleation barriers and atomic diffusion coefficients along inplane and out-of-plane island orientations. Highly anisotropic atomic diffusion constants have been shown to be at the origin of the high aspect ratios of some of the nanostructures. Overall, our study provides a general method for the evaluation of nucleation and coarsening of multiple CSD-derived oxide nanostructures and understanding the shape development by combining thermodynamic and kinetic approaches.

\section{INTRODUCTION}

Nanoscale materials have been investigated for many years due to the reduced dimensionality and new phenomena appearing at their interfaces. Thus, the smart engineering of nanomaterials is fundamental to design novel functional devices with good properties. Bottom-up approaches based on self-assembling and self-organization processes are extremely adequate for the fabrication of nanomaterials, either as thinfilms or nanostructures. They are also envisaged as alternative methods to substitute top-down techniques (e.g. lithography) achieving higher throughput, smaller dimensions and coverage of larger areas homogeneously ${ }^{1-8}$. Self-assembling has been a notably prolific topic for metals and semiconductors with significant contributions aiming to produce interfacial nanostructures through strain engineering $4,8,9$. Complex oxides have also driven much interest due to the very interesting application-oriented properties exhibited such as metallic conductivity, ferroelectricity, ferromagnetism, multiferroicity, 
superconductivity, colossal magnetoresistance, catalytic and photocatalytic activity, ionic conductivity, optics, etc ${ }^{10-24}$. Particularly, cerium dioxide doped with metal ions such as zirconium or gadolinium shows properties such as high dielectric constant and mechanical stability, high ultraviolet absorbance and transparency in the visible/near infrared range, high mechanical stability, catalytic activity and oxygen ionic conductivity that makes it very promising for electro-optical coatings, buffer layer in electronic devices and high-temperature superconducting coated conductors, gas sensors or energy conversion and storage devices ${ }^{25-33}$.

The study of the fundamental principles involved in self-assembling and self-organization of oxides, i.e. nucleation and coarsening, is essential for the fabrication of nanostructured devices with compelling functional properties that usually depend on the particular crystalline orientation ${ }^{34-38}$. These principles are of thermodynamic and kinetic origin, and act as driving forces enabling the growth of nanostructures. However, a large controversy has been generated around the role played by these competing thermodynamic and kinetic mechanisms (e.g. strain relaxation, surface energies, nucleation barriers and atomic diffusion) in the self-assembling and self-organization of epitaxial nanostructures ${ }^{39-42}$. Essentially, the formation of thermodynamically stable nuclei occurs when they grow beyond a critical size overcoming a nucleation barrier. Atomic diffusion mechanisms also contribute to nucleation since they enable the transference of atoms between nuclei, but also the merge of nuclei as whole entities ${ }^{43,44}$. Once nuclei are stable, they grow through coarsening and coalescence which are thermally activated processes ${ }^{45}$. Eventually, nanostructures will reach a stable maximum size which is determined by thermodynamic factors such as the surface energy of island facets, the energy of the interface and strain relaxation mechanisms, but also when the diffusion of atoms to and from nanostructures reaches an equilibrium ${ }^{46-}$ ${ }^{48}$. It is also important to notice that the growth of nanostructures will also depend on the type of system investigated, i.e. an open system where deposition and kinetic evolution of the system occur simultaneously or a closed system where material deposition is finished before the kinetic evolution is enabled. 
Chemical solution deposition (CSD) has appeared among the different processing techniques as an adaptable, low-cost and very powerful ex-situ approach for the bottom-up fabrication of functional oxide architectures with good stoichiometric control. For instance, it has been employed to successfully fabricate a broad range of oxides such as $\mathrm{Pb}(\mathrm{Zr}, \mathrm{Ti}) \mathrm{O}_{3},(\mathrm{Ba}, \mathrm{Sr}) \mathrm{TiO}_{3}, \mathrm{LaNiO}_{3}, \mathrm{Ce}_{1-\mathrm{x}} \mathrm{Gd}_{\mathrm{x}} \mathrm{O}_{2-\mathrm{y}}(\mathrm{CGO})$, ( $\mathrm{La}, \mathrm{Sr}) \mathrm{MnO}_{3}, \mathrm{BiFeO}_{3}$ and $\mathrm{YBa}_{2} \mathrm{Cu}_{3} \mathrm{O}_{7-\mathrm{x}}(\mathrm{YBCO})$ either as polycrystalline and epitaxial films, or in the form of interfacial nanostructures ${ }^{10,35,38,48-55}$. Some attempts have been made to evaluate nucleation and coarsening in closed systems. It is worth mentioning the work done on YBCO and CGO films to determine nucleation barriers and the morphological evolution with the annealing temperature and time ${ }^{38,56}$, and on the study of CGO nanostructures to control the final island morphology, orientation and dimensions with the annealing temperature, time and ambient atmosphere ${ }^{48,57-59}$. Nevertheless, these studies face some limitations since CSD usually uses tubular furnaces which are slow processes. The heating ramps below $0.5^{\circ} \mathrm{C} \mathrm{s}^{-1}$ prevent the independent observation and analysis of nucleation mechanisms which usually depend on temperature and are also strongly linked with the kinetic evolution of the system (i.e. coarsening). Rapid Thermal Annealing (RTA) furnaces are more suitable tools to achieve a precise control over nucleation and growth since they can achieve very fast heating ramps of $20^{\circ} \mathrm{C} \mathrm{s}^{-1}$ and above. Despite the promising advantages of RTA for their use in CSD processing, only few cases have been reported for purposes not related to nucleation and coarsening phenomena. For example, it has been employed in the production of multilayered functional oxide thin-film capacitors and electrodes for electronic devices ${ }^{60}$, ${ }^{61}$ or to avoid the formation of non-desired phases during film growth ${ }^{62}$. Recently, we have shown that RTA can provide an accurate control over epitaxial film growth, and also we used it to investigate the mechanism of orientation-symmetry breaking of CGO nanowires ${ }^{48,54,63}$.

In this work, we will make use of strain-engineering by changing the single crystal substrate and its orientation to study the nucleation and coarsening mechanisms of different CSD-derived gadoliniumdoped ceria $\left(\mathrm{Ce}_{0.9} \mathrm{Gd}_{0.1} \mathrm{O}_{2-\mathrm{y}}\right.$ or $\left.\mathrm{CGO}\right)$ nanostructures as a model system. CGO nanostructures are currently being widely investigated for their use in many applications such as fuel cells and harvesting devices, ${ }^{33}$, 
64, 65 three way emission control catalysts (TWC), ${ }^{66,67}$ gas sensing devices ${ }^{31,68,69}$ or supports in heterogeneous catalysts. ${ }^{70-72}$

These CSD nanostructures are archetypical examples of closed system growth. Hence, nucleation and coarsening are initiated upon ulterior thermal annealing which leads the system to evolve towards thermodynamic equilibrium. Although some works made some attempts to investigate the nucleation and coarsening of CGO nanostructures by means of tubular furnaces ${ }^{57,58}$, to our knowledge, this is the first time that an in-depth study of the coarsening of nanostructures in a closed system is conducted. Instead, all previous kinetic studies of nanostructures published until now have been performed on open systems 40, 73. Particularly, we will grow isotropically-strained heteroepitaxial CGO nanodots (NDs) on (001)oriented $\mathrm{Y}_{2} \mathrm{O}_{3}: \mathrm{ZrO}_{2}(\mathrm{YSZ})$ and $\mathrm{LaAlO}_{3}$ (LAO) substrates, and CGO nanowires (NWs) on (011)LAO substrates. The nucleation and coarsening phenomena exhibited along in-plane and out-of-plane directions will depend on the particular orientation of nanostructures, as well as the presence of low energy facets that can sustain large amounts of oxygen vacancies such as the $\{111\}$ orientation which has been widely investigate for applications in catalysis, gas sensors and ionic conductivity ${ }^{36,74-78}$. Thus, we will perform a detailed study of the formation process of nanoislands from a metal-organic precursor film, and we will evaluate the influence of growth conditions such as the heating ramp, dwell temperature and annealing time on nucleation and coarsening.

\section{EXPERIMENTAL SECTION}

Heteroepitaxial $\mathrm{Ce}_{0.9} \mathrm{Gd}_{0.1} \mathrm{O}_{2-\mathrm{y}}(\mathrm{CGO})$ nanostructures are grown by Chemical Solution Deposition (CSD) methodology. Solutions with a $0.25 \mathrm{M}$ concentration are prepared by mixing Cerium(III) and Gadolinium(III) acetylacetonates (Sigma-Adrich) in propionic acid. The amount of Ce and Gd are, respectively, 1.64 wt. $\%$ and 0.18 wt. $\%$. The solutions are stirred and heated at $50{ }^{\circ} \mathrm{C}$ for 30 min in order to dissolve the metalorganic salts and form cerium - gadolinium propionates. Ultra-diluted solutions with a metal-ion concentration of $0.008 \mathrm{M}$ are prepared by adding proportional amounts of propionic acid. Any remaining impurities are removed by seeping the solution through a $0.2 \mu \mathrm{m}$ filter. Solution deposition is 
done by spin coating at $6000 \mathrm{rpm}, 3000 \mathrm{rpm} \cdot \mathrm{s}^{-1}$ for $2 \mathrm{~min}$ on $5 \times 5 \mathrm{~mm}^{2}(001) \mathrm{Y}_{2} \mathrm{O}_{3}: \mathrm{ZrO}_{2}(\mathrm{YSZ})$, (001) $\mathrm{LaAlO}_{3}(\mathrm{LAO})$ and (011)LAO single crystal substrates thoroughly cleaned by sonication in acetone and methanol. A thermal treatment at $900{ }^{\circ} \mathrm{C}$ during $5 \mathrm{~h}$ under $\mathrm{O}_{2}$ is performed on the substrates before deposition to obtain atomically flat terraces. Afterwards, we perform a pyrolysis at $300^{\circ} \mathrm{C}$ during 10 min in $\mathrm{O}_{2}$ using a tubular furnace in order to completely eliminate the organic compounds and carbon impurities and obtain flat homogeneous nanocrystalline films ${ }^{79}$. Nucleation and kinetic evolution of CGO nanostructures is studied using a Rapid Thermal Annealing (RTA) furnace (AS-Micro from Annealsys) under controlled and stagnated oxygen atmosphere (filled at $51 \mathrm{~min}^{-1}$ for $1 \mathrm{~min}$ ), at temperatures ranging from 800 to $1100{ }^{\circ} \mathrm{C}$ for variable annealing times (0 to $30 \mathrm{~min}$ ), and heating ramps from 0.5 to $20^{\circ} \mathrm{C} \mathrm{s}^{-1}$. The morphology of nanostructures is investigated by atomic force microscopy (AFM) with a 5100 system from Agilent Technologies. The characterization is done in intermittent contact mode using silicon tips of around $10 \mathrm{~nm}$ in diameter, and cantilevers with force constants around $2.8 \mathrm{Nm}^{-1}$. The topography measurements are analyzed with the MountainsMap 7.0 software from Digital Surf.

High resolution and scanning transmission electron microscopy (HRTEM and STEM) observations are carried out to investigate the crystalline structure, orientation, epitaxial relationship with the substrates, morphology and faceting of the nanostructures. Sample preparation is done by mechanical polishing and Ar-ion milling. TEM images are obtained by means of FEI Tecnai F20 and Jeol J2010F systems equipped with field emissions gun operated at $300 \mathrm{kV}$. Lateral resolutions achieved are of $0.14 \mathrm{~nm}$.

Reflection high energy electron diffraction (RHEED) patterns are obtained with a Staib Instruments system using an electron beam of $30 \mathrm{kV}$, a current of $50 \mu \mathrm{A}$ and beam incidence angles with respect to the sample surface below $2^{\circ}$. The investigated incident directions correspond to the $<100>$ and $<110>$ substrate orientations. The diffraction pattern has been simulated with a software based on the kinematic theory of electron scattering.

\section{RESULTS}




\subsection{Nucleation of CGO nanostructures}

\subsubsection{Theoretical formalism for the study of nucleation}

The classical nucleation theory (CNT) is the simplest formalism that describes nucleation events thermodynamically. It states that small clusters of a new phase stabilize after they become large enough to overcome a thermodynamic barrier $G^{*}$. The energy barrier is calculated by assuming a change in the Gibbs free energy $\Delta G$ of the system. In the case of CSD-derived nanostructures, the driving force governing nucleation is the reduction in free energy between the amorphous initial state and the final crystalline one ${ }^{10}$. The most common form of CGO nuclei, as we will see later, is a trapezoidal-prismatic shape (Figure 1) with a height $h$, width $a$ and length $b$, and the lateral facets associated with the width and length are tilted at angles $\delta$ and $\theta$, respectively. Then, the variation of the Gibbs free energy can be written as a function of the effective diameter $S(=\sqrt{a \cdot b})$ 44, 58, 63, 80:

$$
\Delta G(S)=-h S^{2} \Delta G_{v}+\Psi S^{2}+\Gamma S h
$$

where $\Delta G_{v}=\Delta \mu / v$ is the Gibbs free energy per unit volume, $v$ is the unit cell volume, $\Delta \mu(>0)$ is the change in chemical potential or supersaturation between the epitaxial and amorphous/nanocrystalline state. $\Gamma=\left[2\left(\gamma_{b} c \csc \theta+\gamma_{a} c^{-1} \csc \delta\right)-\Phi\left(c \cot \theta+c^{-1} \cot \delta\right)\right], \Psi \equiv \gamma_{t}+\gamma_{i}-\gamma_{s}$ and $\Phi \equiv \gamma_{t}-\gamma_{i}+$ $\gamma_{s}$, with $\gamma_{s}, \gamma_{t}$ and $\gamma_{i}$ being the surface energies of the substrate, top facet of the island, the substrateinterface energy, respectively. $\gamma_{a}$ and $\gamma_{b}$ are the surface energies of the lateral facets, and $c=\sqrt{b / a}$ is the lateral aspect ratio. Parameter values for the different systems are reported in the literature and can be also found in the Supporting Information (Table S1) ${ }^{59,80-82}$. Then, the nucleation barrier as a function of the supersaturation can be written as $48,58,63,80$ :

$$
G^{*}=\frac{-\Gamma^{2} h^{2}}{4\left[-h \frac{\Delta \mu}{v}+\Psi\right]}
$$

At high temperatures, i.e. small undercooling $\left(\Delta T=T_{m}-T\right)$, the supersaturation $\Delta \mu$ is usually described in terms of the latent heat of melting per unit volume $L_{m}$, the melting temperature $T_{m}$ and $v$ the unit cell volume ${ }^{44}$ : 


$$
\Delta \mu=\frac{v L_{m} \Delta T}{T_{m}}
$$

The nucleation rate $d N / d t$ is a kinetic parameter quantifying the number of nuclei that stabilize after overcoming the nucleation barrier. Typically, this process is thermally activated and also depends on atomic diffusion:

$$
\frac{d N}{d t}=\frac{d N_{0}}{d t} \underbrace{\exp \left(-\frac{Q_{n}}{k_{B} T}\right)}_{\text {Atomic mobility }} \underbrace{\exp \left(-\frac{G^{*}}{k_{B} T}\right)}_{\text {Energy barrier }}
$$

The first term considers the effect of atomic mobility which is thermally activated. $Q_{n}$ is the activation energy for atomic diffusion, and $k_{B}$ is the Boltzmann constant. The second term quantifies the number of nuclei that are able to surpass the nucleation barrier. Typically, $Q_{n}$ is assumed to be constant, while $G^{*}$ has a strong dependence with temperature. At the high temperatures investigated the "energy barrier" term dominates the nucleation rate and, hence, it will be the only term considered in our description of nucleation. A more detailed explanation of nucleation and the different regimes can be found elsewhere 80.

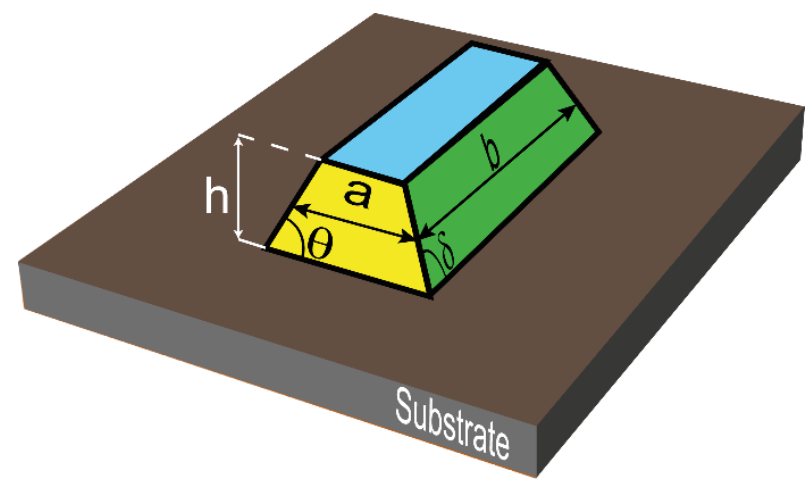

Figure 1. General island shape considered for the theoretical analysis of nucleation.

\subsubsection{Shape of nanostructures}

The shape of nanostructures provides vital information for the study of nucleation and coarsening (i.e. island facets, lattice mismatch, etc). We have evaluated two different systems: CGO isomorphic islands (i.e. nanodots or NDs) grown on (001)YSZ and (001)LAO (Figure 2a,b), and CGO anisotropic parallel 
nanowires (NWs) grown on (011)LAO (Figure 2c). These AFM images show a narrow size distribution with average sizes of approximately $22.6 \pm 2.7 \mathrm{~nm}$ and $21.3 \pm 4.1 \mathrm{~nm}$ in diameter for CGO NDs on (001)YSZ and (001)LAO substrates, respectively, and 21.6 $\pm 2.9 \mathrm{~nm}$ in width and $166.7 \pm 41.9 \mathrm{~nm}$ in length for CGO NWs on (011)LAO substrates. Figure 2d shows the HRTEM characterization of CGO NDs on YSZ. The NDs' shape consists of a truncated pyramid as it has been reported previously for different nanoislands including $\mathrm{CGO}^{48,53,58,83,84}$. The top face of NDs corresponds to the (001)CGO orientation and they grow cube-on-cube on top of the substrate with a relation (001)CGO[100]||(001)YSZ[100] (Figure 2d). The orientation of the lateral facets in Figure $2 \mathrm{~d}$ seems to coincide with (011) planes.
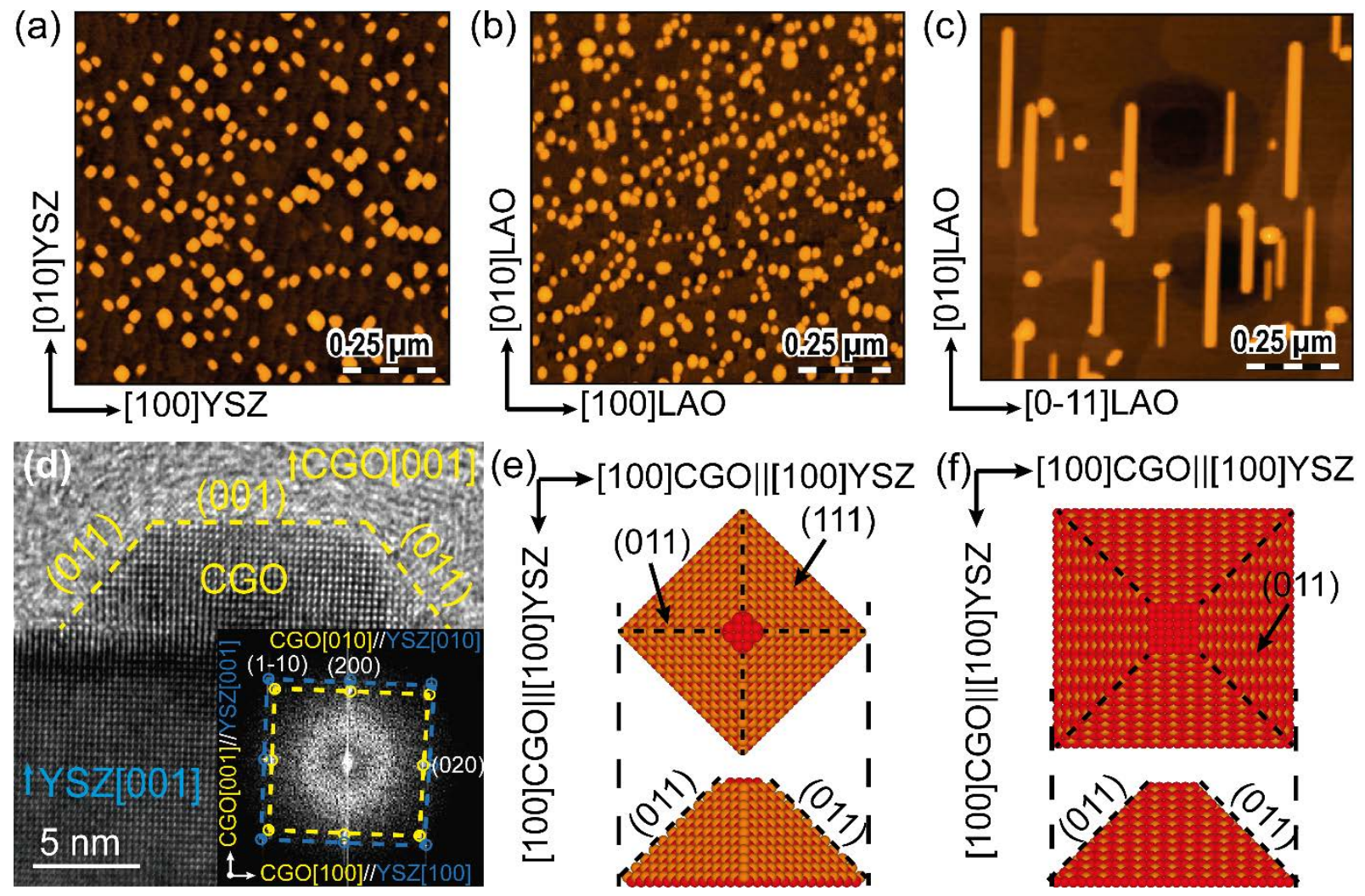

Figure 2. AFM images of CGO nanoislands grown on (a) (001)YSZ, (b) (001)LAO and (c) (011)LAO substrates. All samples have been annealed at $1000{ }^{\circ} \mathrm{C}, 20{ }^{\circ} \mathrm{C} \mathrm{s}^{-1}$ for $15 \mathrm{~min}$ in $\mathrm{O}_{2}$. The average sizes are approximately: (a) $22.6 \pm 2.7 \mathrm{~nm}$ and (b) $21.3 \pm 4.1 \mathrm{~nm}$ in diameter for NDs, and (c) $21.6 \pm 2.9 \mathrm{~nm}$ in width and 166.7 $\pm 41.9 \mathrm{~nm}$ in length for NWs. (d) HRTEM image of CGO NDs on (001)YSZ depicting the parallelepipedic shape of a single island. Inset: power spectrum showing the epitaxial growth and cube- 
on-cube disposition between CGO and YSZ lattices. Solid sphere models of the relation between lateral facets and TEM sample preparation for CGO NDs on YSZ (e) rotated $45^{\circ}$ and (f) without rotation.

However, CGO NDs grow on (001)YSZ with a square-shaped base rotated $45^{\circ}$ from the $<100>$ substrate axes, as opposed to (001)LAO where they grow parallel (Figure 2a and 2b). The TEM specimen was cut parallel to substrate axes, therefore, the lateral facets are the expected (111)CGO planes, i.e. the configuration with lowest surface energy (Table S1), as it is depicted in the solid-sphere models from Figure $2 \mathrm{e}$ and $2 \mathrm{f}^{48,58,83}$. Figure $2 \mathrm{~d}$ also indicates that NDs are fully relaxed on top of (001)YSZ with a compressive lattice mismatch $\varepsilon=\left(a_{Y S Z}-a_{C G O}\right) / a_{C G O} \sim-5 \%\left(a_{Y S Z}=5.145 \AA\right.$ and $\left.a_{C G O}=5.413 \AA\right)$. Figure S1 shows that CGO NDs on (001)LAO have the same island shape, i.e. a truncated pyramid with (111) lateral facets and a (001) top facet. However, for the present case, the CGO lattice is $45^{\circ}$ rotated with respect to the LAO, and thus, the epitaxial relationship is (001)CGO[110]\|(001)LAO[100], as it has been reported before ${ }^{58}$. The lattice mismatch is then $\varepsilon=\left(a_{L A O}-d_{(110) C G O}\right) / d_{(110) C G O} \sim-1 \%$, where $a_{L A O}=3.789 \AA$ and the interplanar distance of (110) planes of CGO is $d_{(110) C G O}=3.827 \AA$. Finally, Figure S2 presents crosssectional HAADF-STEM images of CGO NWs on (001)LAO substrates and their corresponding Fourierfiltered analyses. The NWs have a trapezoidal-like shape with a (011)-oriented top facet and (111) lateral facets along the $[0-11] \mathrm{CGO} \|[100] \mathrm{LAO}$ direction. On the other hand, the lateral facets are (010)-oriented across the $[100] \mathrm{CGO} \|[0-11] \mathrm{LAO}$ direction. The CGO in-plane axes are rotated $90^{\circ}$ with respect to the LAO structure. Hence, a compressive lattice mismatch of $\varepsilon \sim-1 \%$ is obtained in both in-plane directions, as mentioned before ${ }^{48,63}$.

\subsubsection{Heating ramp and temperature dependence of nucleation}

The fast heating ramps obtained with rapid thermal annealing (RTA) furnaces allow tuning of the growth conditions for the separate evaluation of nucleation and coarsening events. Figure S3 shows that our pyrolyzed CGO ultradiluted solutions at $300{ }^{\circ} \mathrm{C}$ for $30 \mathrm{~min}$ on (001)YSZ, (001)LAO and (011)LAO substrates derive in very smooth films with RMS roughness below $0.5 \mathrm{~nm}$. Figure S4 presents the RHEED 
measurements of pyrolyzed CGO films, CGO nanostructures and LAO substrates. These analyses reveal a diffuse halo characteristic of amorphous content (Figure S4a and d). We also observe some faint bright spots arranged on a half circle that can be associated to the substrates (Figure S4b and e) since the depth information is about $5 \mathrm{~nm}$, and the precursor film is $2 \mathrm{~nm}$-thick (Figure S5). Additionally, we see that the halo has some features resembling the typical diffraction patterns of epitaxial CGO nanostructures (Figure S4c and f) which may indicate the presence of nanocrystalline grains. A more detailed study of the local structure has been performed by HRTEM for precursor CGO films pyrolyzed on (001)YSZ, (001)LAO and (011)LAO substrates (Figure S5). We confirm the nucleation of either epitaxial/uniaxial or polycrystalline $\mathrm{CGO}$ grains embedded into a $2 \mathrm{~nm}$ amorphous matrix at temperatures as low as $300{ }^{\circ} \mathrm{C}$. Schwartz and Schneller identified an amorphous metal-oxygen-metal structure in lead/titanium oxides, while the crystallization of other oxides such as barium/strontium titanates may present intermediate crystalline phases before reaching the final phase ${ }^{10,49}$. The structure observed for the CGO system is the final oxide phase which indicates that CGO has a large supersaturation and atomic mobility at low temperatures, and thus, the driving force is considerably large to allow crystallization from an amorphous phase. Roura et al. have also reported the nucleation of ceria thin films at very low temperatures right after decomposition, i.e. at $200{ }^{\circ} \mathrm{C}$ when annealed for $1 \mathrm{~h}{ }^{79}$. The study of nucleation of CGO nanostructures at high temperatures requires to minimize the appearance of CGO nuclei at low temperatures, thus, pyrolysis times of 10 min have been used.

High temperature nucleation studies of CGO NDs on (001)YSZ and (001)LAO, and CGO NWs on (011)LAO substrates have been conducted at $1000{ }^{\circ} \mathrm{C}$ in $\mathrm{O}_{2}$, heating rates of $0.5-20{ }^{\circ} \mathrm{C} \mathrm{s}^{-1}$ and no dwell time (i.e. quench). The AFM images in Figures 3a-c reveal an increase of island dimensions as the heating rate decreases. Analyzing the data extracted from AFM images, we observe that the density of nanoislands (Figure 3d) decreases from approximately 2000 to $1000-500$ islands $\mathrm{nm}^{-2}$ (NDs) and from 1500 to 500 islands $\mathrm{nm}^{-2}(\mathrm{NWs})$ by reducing the heating rate (from 20 to $0.5^{\circ} \mathrm{C} \mathrm{s}^{-1}$ ), whereas the mean volume (Figure 3e) grows from 200-300 to $1200-1400 \mathrm{~nm}^{3}$ (NDs) and from 800 to $4000 \mathrm{~nm}^{3}$ (NWs). These phenomena suggest that coarsening has a large contribution on growth at slow heating rates. In addition, these results 
evidence that all studies about nucleation which have been mostly conducted with conventional thermal treatments (i.e. heating rates $<0.5^{\circ} \mathrm{C} \mathrm{s}^{-1}$ ) are strongly affected by coarsening. Therefore, heating rates of $20{ }^{\circ} \mathrm{C} \mathrm{s}^{-1}$ and above must be employed in order to perform an independent study of nucleation and coarsening. NWs also present a larger volume increase as compared to NDs which indicates larger coarsening. Figure S6 shows the evolution of the different contributions to the mean volume. Figures S6a and S6b show that the equivalent diameter $S$ increases from 10 to $20 \mathrm{~nm}$ when the heating ramp is slowed down from 20 to $0.5{ }^{\circ} \mathrm{C} \mathrm{s}^{-1}$, whereas the height $h$ grows only from 2.5 to $3.5 \mathrm{~nm}$. This indicates a larger contribution of $S$ to the volume increase. For the case of NWs (Figure S6c and d), we see that the height $h$ and the width $a$ have a similar evolution with the heating ramp to that of NDs. Instead, the length $b$ experiences a fast increase in size from 40 to $90 \mathrm{~nm}$ which suggests a stronger contribution of the length to the rise in volume, also confirmed by calculating the lateral aspect ratio $c$ (Figure S6d). We have demonstrated before that the growth of NWs is thermodynamically promoted along their length $48,57,63$, ${ }^{80}$. The non-linear growth of the volume can be related to a variation of the nucleation rate with temperature as we will see later. Despite that, it would be possible that some coarsening is still present. 

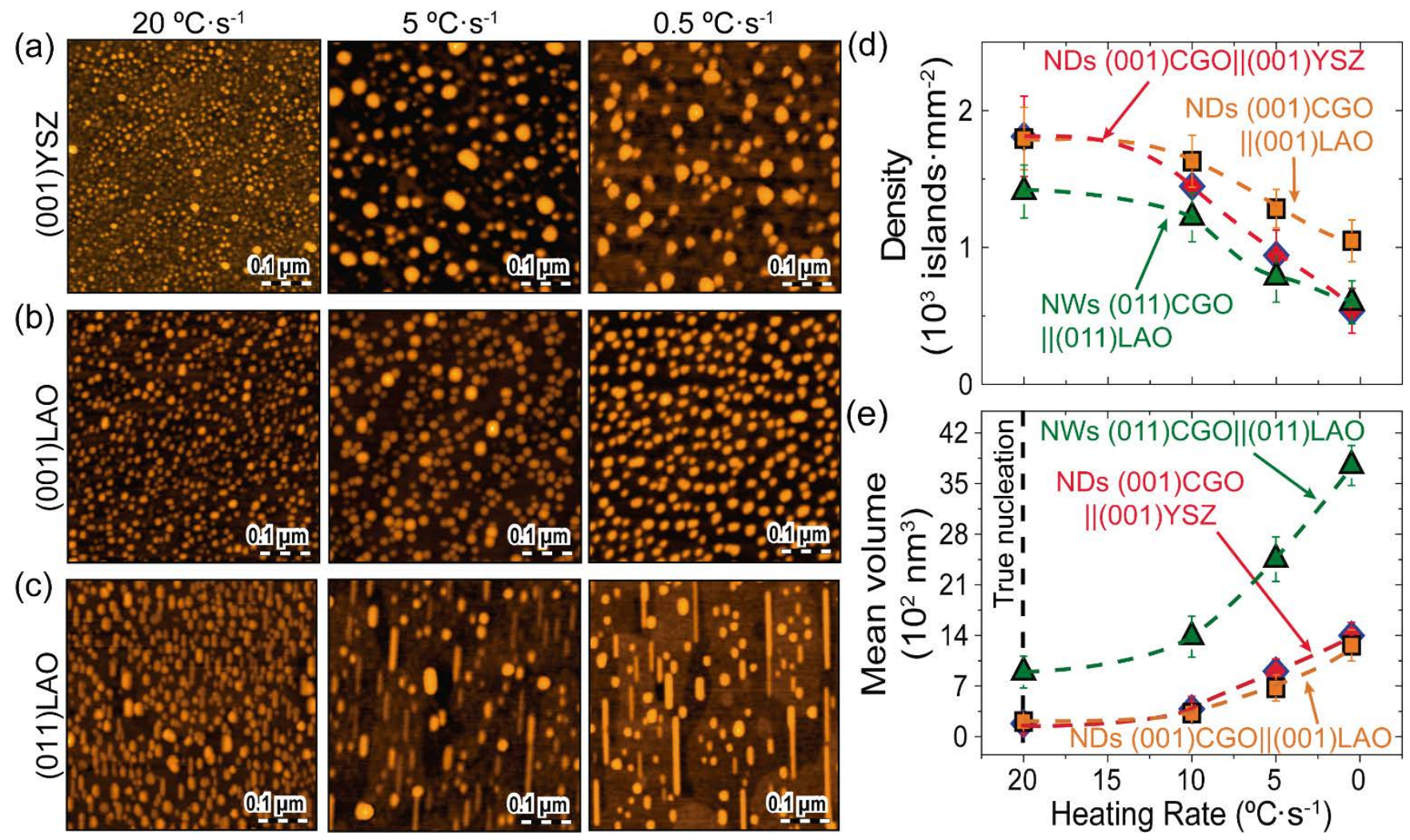

Figure 3. Atomic force microscopy images showing the evolution of nanoislands with the heating rate, for (001)CGO NDs grown on (001)-oriented (a) YSZ and (b) LAO substrates, and (c) (011)CGO NWs grown on (011)LAO substrates. Samples were annealed at $1000{ }^{\circ} \mathrm{C}$ in oxygen without dwell time (quenched samples) and heating rates of 20,5 and $0.5{ }^{\circ} \mathrm{C} \mathrm{s}^{-1}$. Heating rate dependence of the (d) density and (e) mean volume of nanoislands on (001)YSZ (diamonds), (001)LAO (squares) and (011)LAO (triangles) substrates, extracted from AFM images (a-c). The black dashed vertical line indicates the heating rate where the contribution of coarsening is considered to be negligible.

AFM images in Figures 4a-c illustrate the growth of CGO nanoislands with temperature. Quenched samples are annealed on (001)YSZ, (001)LAO and (011)LAO substrates at $20{ }^{\circ} \mathrm{C} \mathrm{s}^{-1}$ in $\mathrm{O}_{2}$ and temperatures between 900 and $1100^{\circ} \mathrm{C}$. These results confirm that temperature indeed has an influence over nucleation rates. This is illustrated more clearly in Figure $4 \mathrm{~d}$ where the density of nanostructures experiences a notable decrease as the annealing temperature rises from 900 to $1100{ }^{\circ} \mathrm{C}$. We have fitted the density of nanoislands considering that the nucleation barrier $G^{*}$ is described by Equation 2 and 3 , 
and that the nucleation rate is defined by the second term in Equation 4. The parameters used for data fitting are $h=1.1 \mathrm{~nm}, v=0.158 \mathrm{~nm}^{3}, T_{m}=2400^{\circ} \mathrm{C}$ and the surface energies reported in Table S1. Then, we can obtain the initial density of nuclei $N_{0}$ and the latent heat of melting per unit volume $L_{m}$ for each system.
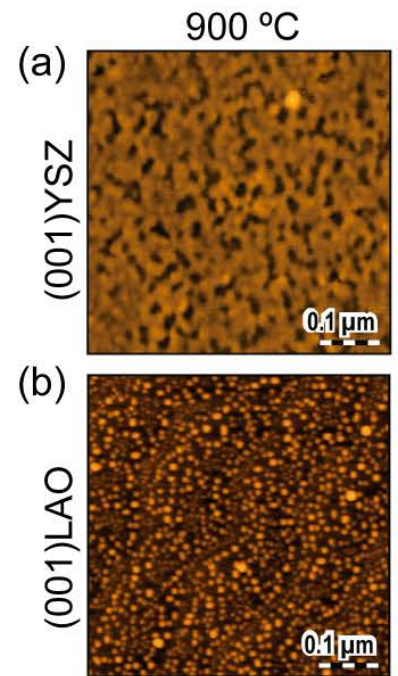

(c)

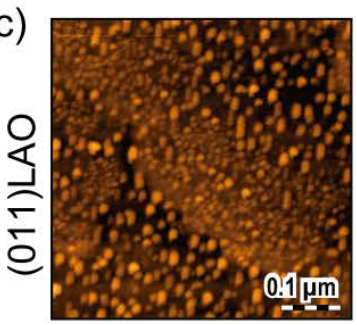

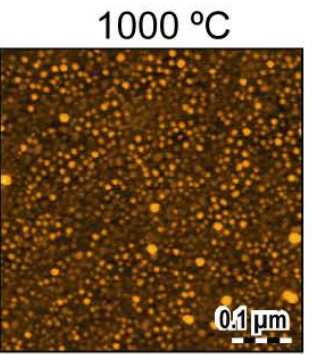
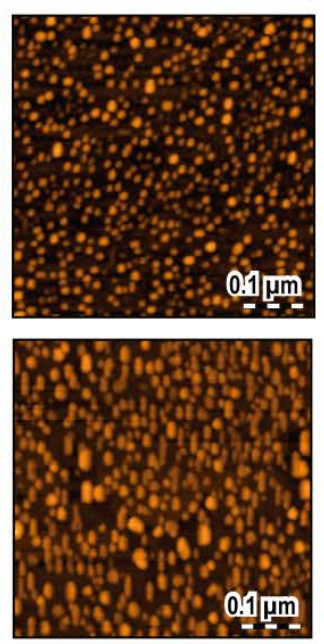

$1100^{\circ} \mathrm{C}$
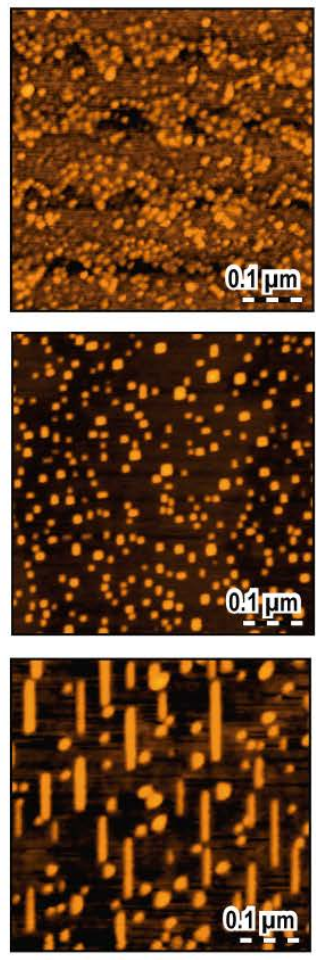

(d)

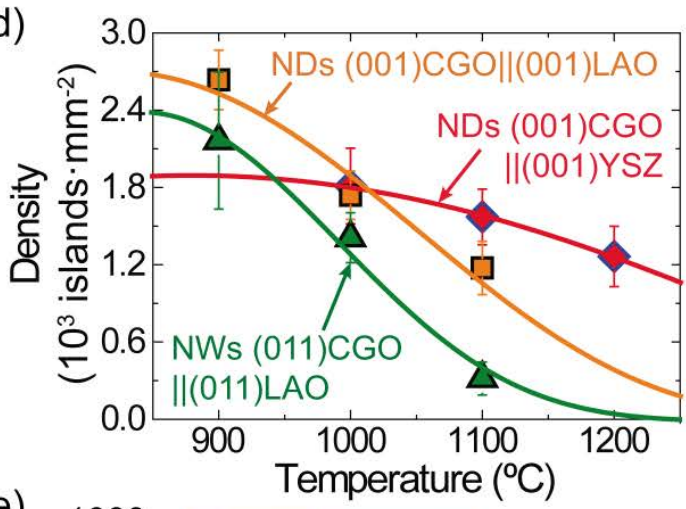

(e)

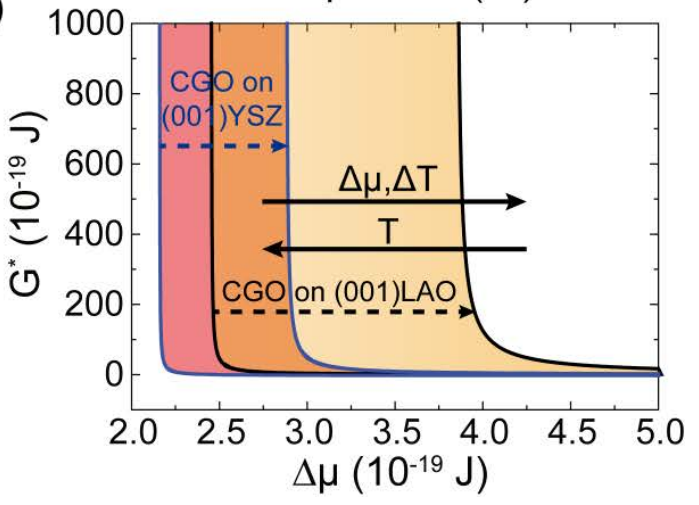

Figure 4. AFM images showing the dependence of nanoislands with temperature for (001)CGO NDs grown on (001)-oriented (a) YSZ and (b) LAO substrates, and (c) (011)CGO NWs grown on (011)LAO substrates. Samples were annealed at $20{ }^{\circ} \mathrm{C} \mathrm{s}^{-1}$ in oxygen and quenched at temperatures of 900,1000 , 1100 and $1200{ }^{\circ} \mathrm{C}$. (d) Temperature dependence of the density of nanostructures for (001)CGO NDs on (001)YSZ (diamonds) and (001)LAO (squares), and (011)CGO NWs on (011)LAO (triangles). Solid lines indicate the fitting of the nucleation rate. Parameters used in the calculations are $h=1.1 \mathrm{~nm}$ and $v=0.158$ $\mathrm{nm}^{3}$. Additional parameters are reported in Table 1. (e) Nucleation barriers for (001)CGO NDs on (001)YSZ and (001)LAO substrates. The dashed arrows indicate the displacement caused by an increase in the interface energy. Values of interface energies employed in the calculations are $\gamma_{i}^{C G O-Y S Z}=0.5 \mathrm{~J}$ $\mathrm{m}^{-2}$ and $\gamma_{i}^{C G O-Y S Z}=1 \mathrm{~J} \mathrm{~m}^{-2}$. 
These values show that (001)CGO NDs grown on (001)LAO and (001)YSZ have similar latent heats, $4.8 \times 10^{9}$ and $5.0 \times 10^{9} \mathrm{~J} \mathrm{~m}^{-3}$ respectively, whereas $N_{0}$ is around two orders of magnitude larger for CGO NDs grown on (001)LAO $\left(4.2 \times 10^{6}\right.$ islands $\left.\mu \mathrm{m}^{-2}\right)$ than for $(001) \mathrm{YSZ}\left(2.7 \times 10^{4}\right.$ islands $\left.\mu \mathrm{m}^{-2}\right)$. For the case of (011)CGO NWs on (011)LAO, $L_{m}$ is around three times lower than that of NDs $\left(1.6 \times 10^{9} \mathrm{~J} \mathrm{~m}^{-3}\right)$, whereas $N_{0}$ is between three and five orders of magnitude larger $\left(5 \times 10^{10}\right.$ islands $\left.\mu \mathrm{m}^{-2}\right)$. It is worth noticing that the values of $L_{m}$ are in agreement with those of other oxide materials such as $\mathrm{La}_{2} \mathrm{O}_{3}, \mathrm{SiO}_{2}, \mathrm{Al}_{2} \mathrm{O}_{3}{ }^{85-}$ ${ }^{87}$. The larger $N_{0}$ for NDs grown on (001)LAO as compared to those on (001)YSZ could point out towards an enhanced driving force for nucleation, and an even bigger driving force for NWs grown on (011)LAO substrates. It could seem that a small variation of the experimental values leads to a large change in the results obtained. However, the derived parameters must have a physical meaning; the nucleation barrier must be positive and increase with temperature which is highly influenced by the value of $L_{m}$. For CGO NWs, we have assumed that the initial nuclei have a lateral aspect ratio $c$ of 1.8-2.5; otherwise, no fitting is possible or $G^{*}$ has negative values.

We can also calculate the nucleation barriers for each system by employing Equation 2 and the parameters from Table S1. Assuming that $T=1000^{\circ} \mathrm{C}$, we calculate that the nucleation barriers from the density of nuclei in Figure $4 \mathrm{~d}$ are $G_{\mathrm{NDs} \|(001) \mathrm{YSZ}}^{*}=(3.8 \pm 0.7) \times 10^{-20} \mathrm{~J}, \quad G_{\mathrm{NDs} \|(001) \mathrm{LAO}}^{*}=(1.1 \pm 0.5) \times 10^{-19} \mathrm{~J}$ and $G_{\mathrm{NWs} \|(011) \mathrm{LAO}}^{*}=(2.5 \pm 0.8) \times 10^{-19} \mathrm{~J}$. These values are very similar and between 3 and 18 times larger than the thermal energy of the system at $1000^{\circ} \mathrm{C}\left(k_{B} T \sim 1.4 \times 10^{-20} \mathrm{~J}\right)$. The lower nucleation barrier of CGO NDs on (001)YSZ of around one order of magnitude as compared to NDs on (001)LAO does not explain why there is a shift to higher temperatures $\left(1000^{\circ} \mathrm{C}\right.$ and above) in the nucleation of $\mathrm{CGO}$ on (001)YSZ when compared to the other systems (Figures 4a-c). We have mentioned before that nucleation rates decrease with temperature. Hence, we have performed a theoretical analysis of the parameters influencing the nucleation barriers. The calculation of nucleation barriers has been done without considering the contribution of strain and interface energies. The contribution of strain is easily addressed by including a term accounting for the misfit strain energy per unit volume $\left(\Delta G_{s t r}\right)$ in Equation 244,63 : 


$$
G_{s t r}^{*}=\frac{-\Gamma^{2} h^{2}}{4\left[-h\left(\frac{\Delta \mu}{v}-\Delta G_{s t r}\right)+\Psi\right]}
$$

CGO NDs grown on (001)YSZ substrates have a larger strain configuration than those grown on (001)LAO $\left(\varepsilon_{(001) \mathrm{CGO} \|(001) \mathrm{YSZ}} \sim-5 \% \text { vs } \varepsilon_{(001) \mathrm{CGO} \|(001) \mathrm{LAO}} \sim-1 \%\right)^{48}$. Large values of strain will contribute increasing the nucleation barrier for a given supersaturation as reported before ${ }^{44}{ }^{63}$. Therefore, the energy required to overcome the nucleation barrier and for the stabilization of nuclei will be higher which means that higher temperatures are needed. On the other hand, nucleation barriers are often calculated considering that the interface energy is negligible $\left(\gamma_{\mathrm{i}}=0 \mathrm{~J} \mathrm{~m}^{-2}\right.$ in Equation 2). Despite that, the interface energy may also contribute shifting nucleation barriers. We can assume that the interface energy between CGO and YSZ, i.e. both have fluorite structures, will be much lower than CGO on LAO (fluorite/perovskite). Figure 4e presents the results of the calculation of nucleation barriers for CGO on (001)YSZ and (001)LAO substrates with interface energies from $0 \mathrm{~J} \mathrm{~m}^{-2}$ to 0.5 and $1 \mathrm{~J} \mathrm{~m}^{-2}$, respectively. We observe that the nucleation barrier of CGO on (001)YSZ is shifted to lower values of supersaturation $\left(\Delta \mu \sim \Delta T=T_{m}-T\right)$ as compared to $\mathrm{CGO}$ on (001)LAO which supports our experimental results, i.e. the nucleation temperature must be higher. If we consider the contribution of interface energies $\left(\gamma_{i}^{Y S Z}=0.5 \mathrm{~J}\right.$ $\mathrm{m}^{-2}$ and $\gamma_{i}^{L A O}=1 \mathrm{~J} \mathrm{~m}^{-2}$ ), we see that nucleation barriers are displaced to large values of supersaturation (lower temperatures). Given that the interface energy of CGO on (001)LAO must be much larger than on (001)YSZ, the nucleation barrier will be shifted to even higher values of $\Delta \mu$. Hence, lower temperatures are required to nucleate $\mathrm{CGO}$ NDs on $\mathrm{LAO}$ substrates.

These results seem to confirm that we have successfully separate nucleation from coarsening. Nevertheless, the contribution of coarsening cannot be completely discarded due to the large atomic diffusion at such high temperatures. In fact, Meixner et al. developed a theoretical work which suggests that kinetic mechanisms rule over thermodynamics in nucleation, thus, leading to an increase of nanostructure dimensions with temperature ${ }^{39}$. Figure S7 shows the variation of volume with the heating rate at $1000{ }^{\circ} \mathrm{C}$ plotted together with the volume variation with temperature at $20^{\circ} \mathrm{C} \mathrm{s}^{-1}$ for CGO NDs and 
NWs. The volume increase with temperature for CGO NDs may indicate that some coarsening is still present. Despite that, these values are smaller than the ones obtained using slower heating ramps $\left(<10^{\circ} \mathrm{C}\right.$ $\mathrm{s}^{-1}$ ). The only case where coarsening may have a large contribution is that of CGO NDs grown on (001)YSZ at temperatures above $1100^{\circ} \mathrm{C}$ (Figure S7a). Figure S7c shows that CGO NWs follow a trend equivalent to that of NDs. However, the influence of coarsening seems very important at temperatures above $1000^{\circ} \mathrm{C}$, given that the mean volume of NWs is similar to that of heating ramps slower than $5^{\circ} \mathrm{C}$ $\mathrm{s}^{-1}$. Therefore, we can consider that the influence of coarsening in nucleation is limited to high temperatures and slow heating rates.

\subsection{Kinetic evolution of CGO nanostructures}

\subsubsection{Theoretical formalism for the study of coarsening}

Crystal growth is a thermodynamically and kinetically governed phenomenon and it has been a long standing issue in epitaxial growth of nanostructures to unveil the role of thermodynamic versus kinetics 39,40 . As we have described before, nucleation is a kinetically governed phenomenon with energy barriers of thermodynamic origin. Once nuclei are stabilized, they enlarge spontaneously at expenses of other nuclei leading to the uniform size characterizing self-assembly principles ${ }^{45}$. This is again a thermodynamic driven process governed by kinetic parameters such as atomic diffusion constants. This phenomenon is called Ostwald ripening. It is driven by the decrease in the energy of a system due to the reduction of the overall surface-to-volume ratio ${ }^{88,89}$; hence, promoting the formation of large crystallites from small ones by atomic diffusion, also known as coarsening. It is however, possible that the nanostructures display anisotropic diffusion constants and so their shape aspect can also be determined by kinetic effects. Coalescence is another relevant growth mechanism which can be static, when two nuclei grow independently by Ostwald ripening and, eventually, merge into an individual entity ${ }^{90}$; or dynamic when two nuclei merge after they diffuse into each other ${ }^{91,92}$.

The kinetic mechanisms of grain growth were described long time ago by Burke and Turnbull for polycrystalline materials in isothermal conditions. Assuming that energy minimization and the reduction 
of the surface area through atomic diffusion are the mechanisms driving grain boundary reconstruction, grain growth is described by a growth rate $d S / d t$ with a general solution ${ }^{93}$ :

$$
S^{n}-S_{0}^{n}=k_{n} t
$$

where $S$ and $S_{0}$ are the average and initial grain diameter, $k_{n}=n M \gamma S^{n-2}$ is a material constant, $M$ is the grain boundary mobility, $\gamma$ the grain boundary energy, and $n$ is the growth exponent with values between 2 and 4. Even though this formulism has been used in many cases, it is inadequate to analyze grain growth in the nanometer range where the dependence of atomic diffusion with the grain size and crystalline defects often limit the movement of grain boundaries ${ }^{89,94}$. The time evolution of isothermal grain coarsening $S(t)$, as well as epitaxial crystallization and nanoislands' growth, can be described by a self-limiting model of the form ${ }^{56,95-98}$

$$
S(t)-S_{0}=\left(S_{\max }-S_{0}\right)\left[1-\exp \left(-\frac{t}{\tau}\right)\right]
$$

where $S_{0}$ and $S_{\max }$ are respectively the initial and final dimensions, and $\tau$ is the characteristic relaxation time (at $0.63 S_{\max }$ ). The diffusion coefficient at a fixed temperature $D_{T}$ is then calculated with the expression:

$$
D_{T}=\frac{\left(S_{\max }-S_{0}\right)^{2}}{4 \tau}
$$

The temperature dependence of the diffusion can be obtained by considering a thermally-activated process and fitting the data with an Arrhenius law $56,95-98$ :

$$
D=D_{0} \exp \left(\frac{-Q_{d}}{k_{B} T}\right)
$$

where $D_{0}$ is a pre-exponential factor that depends on parameters such as the lattice constant and other geometrical constants, $Q_{d}$ is the activation energy for diffusion and $k_{B}$ is the Boltzmann constant. $D_{0}$ and $Q_{d}$ are both assumed constant. As we will see in the next section, the kinetic evolution of all the nanostructures investigated here can be properly described using the self-limiting model described by Equation (7). 


\subsubsection{Thermally-activated coarsening of CGO nanostructures}

A great discussion has been created in relation to whether thermodynamic or kinetic mechanisms control the formation of nanostructures. We have reported in previous works that coarsening of nanostructures is thermodynamically promoted due to a reduction in the total energy of the systems $48,57,59,63$. Despite that, diffusion mechanisms also play an important role in the definition of nanoisland dimensions ${ }^{99}$. Systems based on the deposition of chemical solutions have the final amount of material already deposited from the beginning of the growth process. Hence, it should be expected that our nanostructures behave close to a system in thermodynamic equilibrium as opposed to physical methods such as pulsed laser deposition where material is being deposited continuously.

Figures 5a-c show AFM images of the size evolution with time of (001)CGO NDs grown on (001)LAO substrates after heating at $20^{\circ} \mathrm{C} \mathrm{s}^{-1}$ in $\mathrm{O}_{2}$, temperatures between 900 and $1100{ }^{\circ} \mathrm{C}$, and dwell times from 0 to 15 min. Equivalent measurements have been conducted for (001)CGO NDs on (001)YSZ (Figure S8). A detailed analysis of the time evolution of the equivalent diameter $S$ and height $h$ can be extracted from these AFM images (Figures 5d, 5e and S9). Clearly, both $S$ and $h$ increase with time describing a saturation behavior which is more pronounced at high annealing temperatures. Particularly for (001)CGO NDs on (001)LAO (Figures $5 \mathrm{~d}$ and 5e), we observe that $S$ increases from 5-12 $\mathrm{nm}$ to $20-25 \mathrm{~nm}$ depending on the annealing temperature, whereas $h$ grows from 2-3.5 $\mathrm{nm}$ to $5-7 \mathrm{~nm}$. Figure $\mathrm{S} 9$ shows that $S$ and $h$ evolve respectively with the annealing time from approximately 10 to $22 \mathrm{~nm}$ and from 2.5 to $5-6 \mathrm{~nm}$ for (001)CGO NDs on (001)YSZ. These results indicate that CGO NDs always reach an equilibrium maximum size, as we predicted before using thermodynamic calculations ${ }^{48,59}$. However, atomic diffusion increases with temperature as we will see later, and thus, the kinetic growth process is speeded up at high temperatures. The height-to-diameter ratios $h / S$ do not show a clear evolution with the annealing time and temperature with values between 0.25 and 0.35 for CGO NDs grown on LAO and between 0.18 and 0.25 on YSZ substrates (Figure S10). However, these results indicate that the lateral dimensions of NDs are always larger than their height $(h / S<1)$. The formation of nanostructures is usually promoted in highly 
strained systems since they are less restricted in the in-plane direction as compared to films, and thus, they are more free to adapt their structure leading to reduced strain. From a thermodynamic perspective, a stronger relaxation of the system should be obtained for nanostructures with $h / S>1$ due to the presence of less strained regions ${ }^{8}$. However, kinetic mechanisms have a large influence over their growth. Tersoff and Tromp suggested that there is a tradeoff between strain relaxation and surface energy which leads to nanostructures with larger in-plane dimensions ${ }^{46}$. Essentially, under certain conditions it is more favorable for atoms to diffuse into the island edges instead of diffusing to the top island facet, hence, enhancing strain relaxation and decreasing the overall system energy despite the extra surface energy. This phenomenon occurs in open systems where atomic diffusion is faster on the substrate than on nanostructures. It also seems to be the same case for closed CSD systems where atoms are already deposited on the substrate surface prior to the formation of nanostructures (Figure S3).
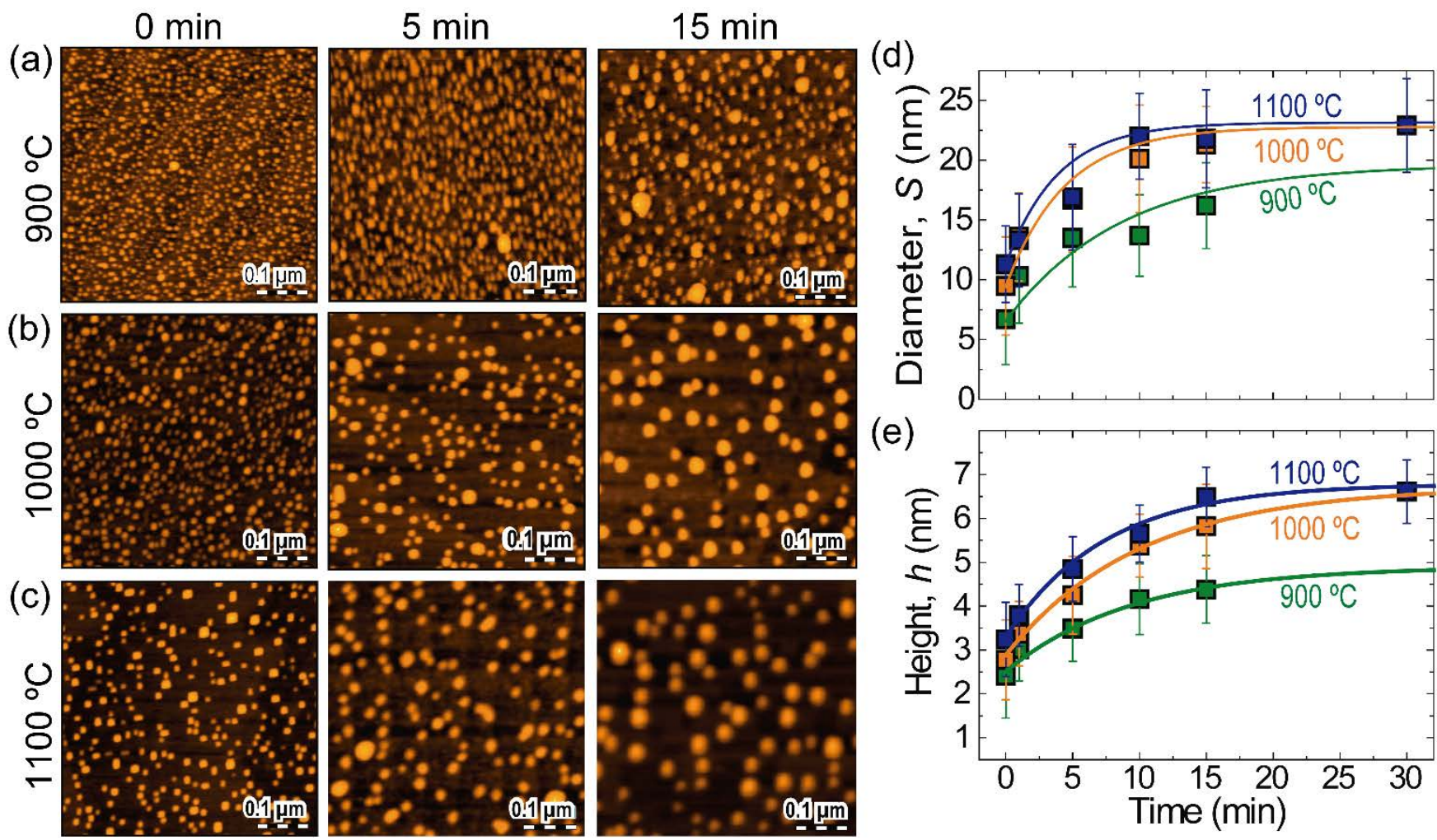

Figure 5. AFM images of the coarsening evolution with time of (001)CGO NDs grown on (001)LAO substrates at $20{ }^{\circ} \mathrm{C} \mathrm{s}^{-1}$ in $\mathrm{O}_{2}$, and (a) 900, (b) 1000 and (c) $1100{ }^{\circ} \mathrm{C}$. Temperature and time dependence of (d) the equivalent diameter $S$ and (e) the height $h$ of CGO NDs on (001)LAO extracted from AFM images $(\mathrm{a}-\mathrm{c})$. 
Figure 6 shows equivalent analyses of the evolution with time of the in-plane and out-of-plane dimensions of (011)CGO NWs grown on (011)LAO substrates. The width of NWs $a$ increases in size from 12-16 nm to $16-22 \mathrm{~nm}$ depending on the annealing temperature (Figure 6d), while the height $h$ goes from 1-2 $\mathrm{nm}$ to 2-7 nm (Figure 6e). Both dimensions show an equivalent saturation behavior to that described for CGO NDs. Equivalently, the length of NWs $b$ grows from 25 to $100 \mathrm{~nm}$ at $900{ }^{\circ} \mathrm{C}$, showing a similar saturation behavior. However, larger temperatures $\left(1000-1100{ }^{\circ} \mathrm{C}\right)$ show growth in the length from $50-75 \mathrm{~nm}$ to around $250 \mathrm{~nm}$ which seems to point out towards either longer saturation times or continuous enlargement of NWs. This is more clearly seen in Figure S11a where the length-to-width of NWs b/a increases with the annealing time and temperature. The height-to-width ratio $h / a$ slightly increases with the annealing time and temperature from approximately 0.1 to 0.3 (Figure S11b), whereas the height-to-length ratio $h / b$ (Figure S11c) seems constant with values around 0.02-0.04. Similarly to NDs, the in-plane dimensions are larger than out-of-plane ones which again could be due to faster diffusion of atoms to the lateral facets as compared to the top facet. It is worth noticing that NWs' elongation is in agreement with previous theoretical studies where we have demonstrated that coarsening of NWs along their length is thermodynamically driven by the continuous reduction of the system's energy ${ }^{63}$. Therefore, NWs' elongation seems to be a kinetically-driven thermodynamic process. 

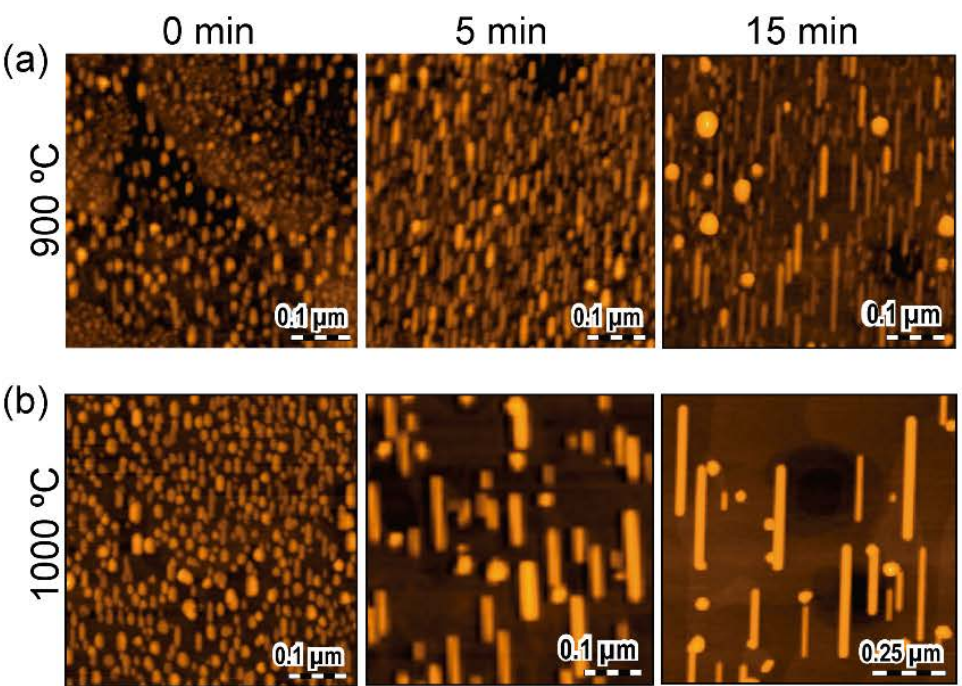

(c)
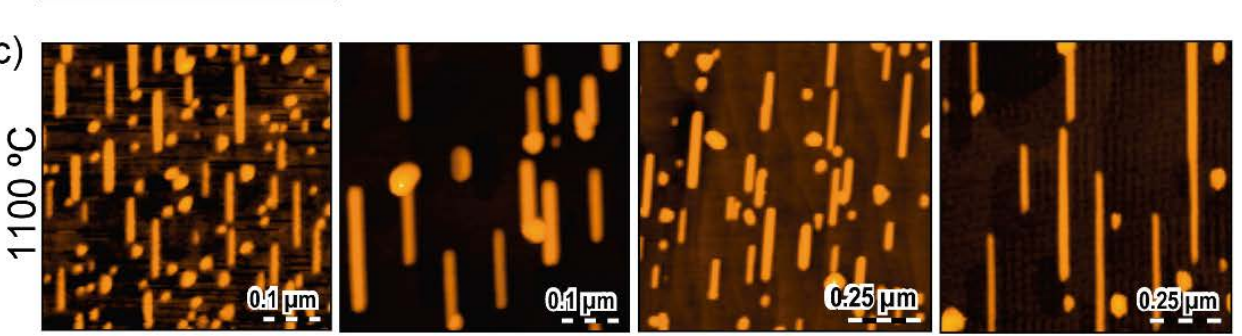

(e)
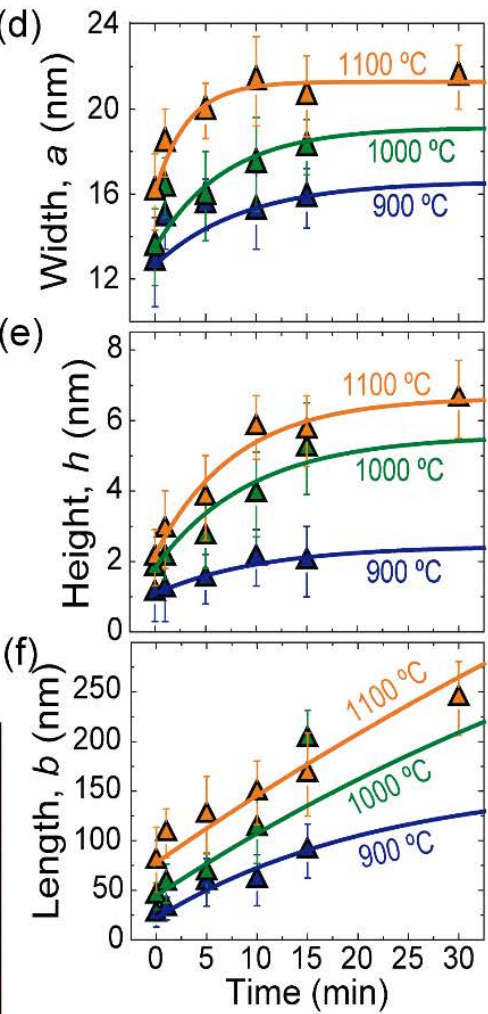

Figure 6. AFM images of the influence of coarsening after heating at $20^{\circ} \mathrm{C} \mathrm{s}^{-1}$ for $(011) \mathrm{CGO} N W s$ grown on (011)LAO substrates at (a) 900, (b) 1000 and (c) $1100{ }^{\circ} \mathrm{C}$ in $\mathrm{O}_{2}$. Time dependence of the (d) width $a$, (e) height $h$ and (f) length $b$ of (011)CGO NWs on (011)LAO substrates, extracted from AFM images (ac).

A self-limited growth model (section 3.2.1) can be used to describe the saturation coarsening behavior observed. Figure 7 a presents the dependence with temperature of the diffusion coefficients for the inplane and out-of-plane directions $\left(D_{\|}^{N D s}\right.$ and $\left.D_{\perp}^{N D s}\right)$ extracted from Equation 7 and 8 . We can see that atomic diffusion increases with temperature, as expected from a thermally-activated kinetic process. In addition, atomic diffusion is around one order of magnitude faster along the in-plane direction as compared to the out-of-plane one. Since atomic diffusion follows an Arrhenius-like thermally-activated process (Equation 9), we can extract precise values of in-plane and out-of-plane activation energies $\left(Q_{\|}^{N D s}\right.$ and $\left.Q_{\perp}^{N D s}\right)$ and pre-exponential factors $\left(D_{0, \|}^{N D s}\right.$ and $\left.D_{0, \perp}^{N D s}\right)$ from the corresponding Arrhenius plots (Figure 7b). The in-plane activation energy for island coarsening is slightly larger than the out-of-plane 
counterpart $\left(Q_{\|}^{N D s}=0.81 \pm 0.13 \mathrm{eV}\right.$ and $\left.Q_{\perp}^{N D s}=0.69 \pm 0.11 \mathrm{eV}\right)$, indicating that a little more energy is required to activate in-plane diffusion. These values are around 6-7 times larger than the thermal energy $k_{B} T(\sim 0.1$ $\mathrm{eV}$ at $1000^{\circ} \mathrm{C}$ ) and smaller than those reported in the literature for polycrystalline CGO films produced by spray pyrolysis and PLD, and YSZ films produced by precipitation 56, 96, 100, 101. These low energy values seem to indicate that surface or grain boundary diffusion could be the main mechanisms contributing to coarsening since these processes typically yield energy values between 0.1 and $1 \mathrm{eV}^{98}$. On the other hand, the in-plane pre-exponential factor $\left(D_{0, \|}^{N D s}=(6.0 \pm 0.1) \times 10^{-16} \mathrm{~m}^{2} \mathrm{~s}^{-1}\right)$ is two orders of magnitude larger than the out-of-plane value $\left(D_{0, \perp}^{N D s}==(9.8 \pm 0.1) \times 10^{-18} \mathrm{~m}^{2} \mathrm{~s}^{-1}\right)$. Since activation energies are rather similar, it seems that the difference in pre-exponential factors illustrate the larger atomic mobility along the in-plane direction. Activation energies are related to barriers for atomic jumps, i.e. diffusion. Therefore, the pre-exponential factors $D_{0}$ could be related to the number sites available for diffusion. $D$ values reflect basal diffusion stress barriers that control adatom diffusion and are also essential to keep a narrow size distribution ${ }^{45,99}$. In this case, the results obtained may indicate a faster surface mobility in the direction parallel to the substrate than the atomic diffusion along the lateral facets of nanostructures heightwise, and also a faster mobility of atoms on the substrate than on nanostructures due to the high density of adatoms, i.e. surface atoms ${ }^{46,102}$. Nevertheless, several additional aspects should be considered to influence growth of nanodots. We mentioned and demonstrated before that there exists a thermodynamic driving force promoting growth until a stable size is achieved ${ }^{48,59}$. This driving force (or growth rate) decreases as NDs' dimensions approach the optimum size and it also highlights the role of island facets on promoting self-limiting growth and a narrow distribution of sizes independently of the lattice mismatch ${ }^{99}$. In addition, it is very likely that the proximity of nuclei at early stages of growth contributes to a faster atomic diffusion than at later phases. Finally, it has been reported that the presence of a repulsive biaxial strain field stimulates nucleation of NDs while it hinders their growth as they become bigger $^{103}$. 

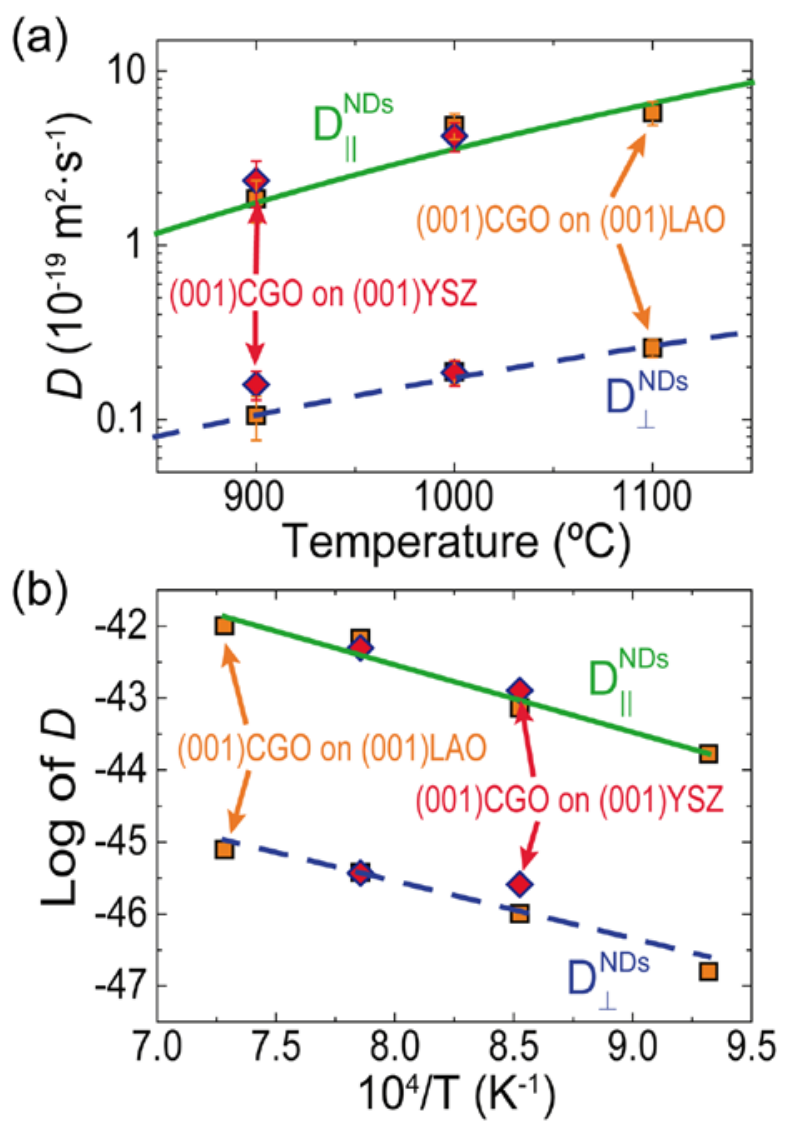

Figure 7. Temperature dependence of the (a) in-plane and out-of-plane diffusion coefficients $\left(D_{\|}^{N D s}\right.$ and $D_{\perp}^{N D s}$ ). (b) Arrhenius plots of $D_{\|}^{N D s}$ and $D_{\perp}^{N D s}$. (001)CGO NDs on (001)YSZ and (001)LAO are represented by diamonds and squares, respectively.

In the case of CGO NWs, the analysis of width, length and height diffusion coefficients $\left(D_{\|, a}^{N W s}, D_{\|, b}^{N W s}\right.$ and $D_{\perp}^{N W s}$ ) in Figure 8a also shows the expected rise with temperature associated with a thermallyactivated diffusion process. We can see that the range of $D_{\|, a}^{N W s}$ and $D_{\perp}^{N W s}$ is equivalent to that of NDs inplane and out-of-plane diffusion coefficients (Figure 7a) as expected since they have similar self-limited behaviors. Thus, the same mechanisms as for NDs should be applicable. On the other hand, $D_{\|, b}^{N W s}$ is between two and three orders of magnitude larger than previous diffusion coefficients; clearly illustrating the anisotropic growth of NWs. The mechanisms leading to such anisotropy will be discussed next. From the Arrhenius plots in Figure 8b, we can determine that the activation energies for width, length and height are respectively $Q_{\|, a}^{N W s}=1.06 \pm 0.38 \mathrm{eV}, Q_{\|, b}^{N W s}=1.38 \pm 0.56 \mathrm{eV}$ and $Q_{\perp}^{N W s}=1.63 \pm 0.56 \mathrm{eV}$. Although these 
values are quite similar between them, they indicate that less thermal energy is required to activate atomic diffusion along the $a$ direction. Again, surface or grain boundary diffusion seems to be the main mechanism due to the low activation energies of $\sim 1 \mathrm{eV}$. In this case, the activation energies are between 9 and 15 times larger than the thermal energy $k_{B} T$ and around two times the activation energies of NDs. Considering this, we should expect a slightly delayed diffusion of NWs as compared to NDs. However, the pre-exponential factors of NWs width, length and height $\left(D_{0, \|, a}^{N W s}=(3.6 \pm 0.2) \times 10^{-16} \mathrm{~m}^{2} \mathrm{~s}^{-1}\right.$, $D_{0, \|, b}^{N W S}=(5.2 \pm 0.6) \times 10^{-12} \mathrm{~m}^{2} \mathrm{~s}^{-1}$ and $D_{0, \perp}^{N W s}=(3.4 \pm 0.5) \times 10^{-14} \mathrm{~m}^{2} \mathrm{~s}^{-1}$, respectively $)$ are significantly larger than those of NDs, compensating for the higher $Q$ values and indicating that the driving force for atomic diffusion is larger for NWs as compared to NDs. In addition, the higher value of $D_{0, \|, b}^{N W s}$ in relation with $D_{0, \| l, a}^{N W s}$ and $D_{0, \perp}^{N W s}$ explains why atomic diffusion coefficients are around three times larger, and the faster coarsening and bigger dimensions along the $b$ direction. Atomic diffusion also has a large influence at early stages of NWs' growth. However, we reported before that they may have no thermodynamic restriction towards unlimited growth ${ }^{63}$. In addition, Li et al. mentioned that nanostructures with an anisotropy in their shape and surface energies should grow along the lower surface energy facet in order to reduce the energy of the system ${ }^{47}$. In the case of CGO, the $\{111\}$ lateral facets have a lower surface energy than $\{001\}$ facets as reported in Table S1 and Refs. ${ }^{59,63}$. We demonstrated previously that the elongation of NWs occurs along the direction with $\{111\}$ facets as shown in Figure S2 and Refs. ${ }^{57,58,63 .}$ Consequently, the origin of such anisotropic in-plane diffusion constants could be the result of the dissimilar island facets and their surface energies. Overall, the kinetic evolution of CGO nanostructures seems to direct the growth process towards thermodynamic equilibrium as expected from a closed system ${ }^{8}$. The internal redistribution of adatoms is driven by the need to minimize the surface energy. Thus, we conclude that shape evolution is driven by thermodynamics but mastered by kinetic effects, i.e. enhanced atomic mobility along specific directions (strain is not the driving force since it is isotropic in the systems considered). 

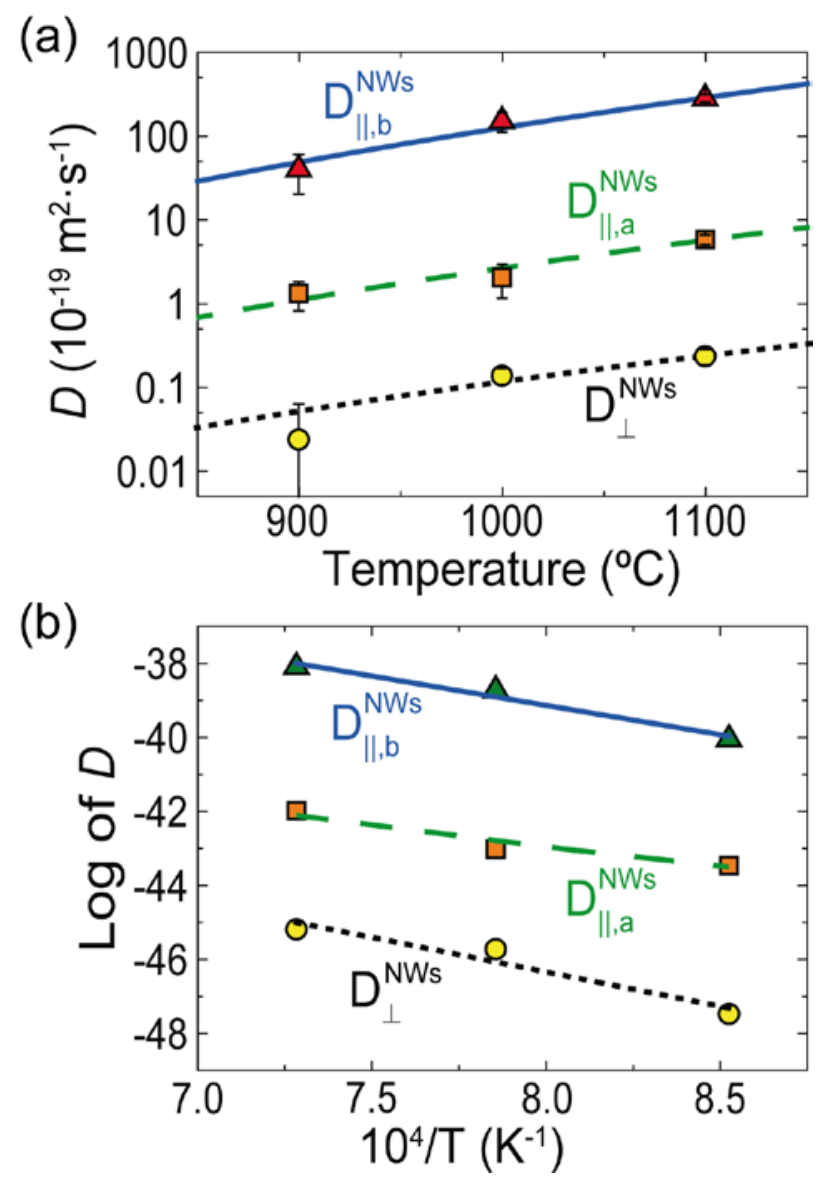

Figure 8. (a) Dependence with temperature of the diffusion coefficients of (011)CGO NWs on (011)LAO substrates along their width $\left(D_{\|, a}^{N W s}\right)$, length $\left(D_{\|, b}^{N W s}\right)$ and height $\left(D_{\perp}^{N W s}\right)$. (b) Arrhenius plots of $D_{\|, a}^{N W s}$, $D_{\|, b}^{N W s}$ and $D_{\perp}^{N W s}$

\section{CONCLUSIONS}

We have investigated the nucleation and coarsening of different CGO nanostructures. In particular, we have shown that different shapes can be obtained by changing the strain relation between the CGO structure and the substrate. Essentially, isotropic CGO NDs have been grown on (001)-oriented YSZ and LAO substrates with biaxial in-plane strains of $-5 \%$ and $-1 \%$, respectively, and with the (001)CGO[100]||(001)YSZ[100] and (001)CGO[100]||(001)LAO[100] epitaxial relationships between NDs and substrate. On the other hand, anisotropic CGO NWs have been obtained on (011)-oriented LAO substrates with a biaxial in-plane strain of $-1 \%$. The epitaxial relationship with the substrate in this case is $(011) \mathrm{CGO}[100] \|(011) \mathrm{LAO}[0-11]$. 
We determined that nucleation and even epitaxy of CGO nanograins (i.e. the final oxide phase) is possible at temperatures as low as $300{ }^{\circ} \mathrm{C}$ which is rarely the common observations in CSD oxide growth. We also demonstrated, taking advantage of fast RTA furnaces, that it is possible to study nucleation and coarsening independently. The use of fast heating rates in the range of $20^{\circ} \mathrm{C} \mathrm{s}^{-1}$ allows us to reduce the contribution of coarsening in nucleation, as compared to the slow heating rates achieved using conventional thermal treatments $\left(<0.5^{\circ} \mathrm{C} \mathrm{s}^{-1}\right)$. The evolution of nanoislands with temperature has also shown a small increase in sizes, although it is not comparable to the growth experienced by decreasing the heating rate. We observed an experimental shift in the nucleation temperature for CGO NDs grown on (001)LAO and (001)YSZ. Thermodynamic calculations reveal that the strain energy shifts the nucleation barriers to smaller values of supersaturation, i.e. larger temperatures. Thus, the larger lattice mismatch of $-5 \%$ between CGO and YSZ structures as compared to CGO on LAO ( $\varepsilon_{\sim-1 \%}$ ) delays nucleation to higher temperatures. In addition, the lower interface energy of CGO on YSZ contributes to lower supersaturation values, and thus, higher nucleation temperatures.

The coarsening of nanostructures has been described with a self-limited growth model. We demonstrated that it is driven by a thermally-activated diffusion process, i.e. atomic diffusion increases with temperature, that leads to thermodynamic equilibrium. For CGO NDs, we have determined that the activation energies for atomic diffusion are quite similar for in-plane and out-of-plane directions, and indicate that surface diffusion may be the main coarsening mechanism. Atomic diffusion is faster along the in-plane direction because is thermodynamically more favorable for atoms to diffuse into island edges instead of the top facet in order to enhance strain relaxation. Thermodynamics also rules the growth of NDs until a stable island dimension, i.e. narrow size distribution, is achieved. The activation energies for atomic diffusion for CGO NWs are larger than those of NDs. Despite that, the coarsening of CGO NWs shows equivalent diffusion trends along their width and height. Thus, similar self-limited diffusion mechanisms control growth along these directions. The coarsening along NWs' length is orders of magnitude faster than the other directions. The large driving force along NWs length is likely promoted by the dissimilar surface energies, and the enhanced growth along the direction with the lowest energy 
$\{111\}$ lateral facets. Overall, the growth of CGO nanostructures is controlled by thermodynamics, but mastered by kinetic effects.

The results obtained demonstrate that we can successfully investigate nucleation and coarsening separately thanks to the fast heating ramps achieved with rapid thermal annealing furnaces. In addition, they provided a better comprehension of the mechanisms involved in the growth of CGO nanostructures, giving more knowledge on the still insufficiently understood nucleation and coarsening processes. The thorough investigation and theoretical calculations employed can also be systematically used for the study of nucleation and growth of more complex systems where a larger number of nuclei are involved such as epitaxial oxide thin films. It is also worth mentioning the use of the proposed methodology for the growth of epitaxial oxide nanostructures on very different substrates such as silicon which is used for microelectronic applications, given that the precise control offered by RTA furnaces.

\section{ASSOCIATED CONTENT}

Supporting Information. Parameters used in thermodynamic calculations, additional TEM characterization of nanostructures, AFM, RHEED and TEM characterization of precursor films, additional data for the evolution of nanostructure dimensions with the heating rate and time.

\section{AUTHOR INFORMATION}

\section{Corresponding Author}

*Dr. Albert Queraltó

ICMAB - CSIC, Campus UAB, 08193 Bellaterra, Catalonia, Spain

Tel. +34935801853

E-mail: albert.queralto.lopez@gmail.com 


\section{Author Contributions}

The manuscript was written through contributions of all authors. All authors have given approval to the final version of the manuscript.

\section{ACKNOWLEDGMENT}

We acknowledge financial support from Spanish Ministry of Economy and Competitiveness through the "Severo Ochoa" Programme for Centres of Excellence in R\&D (SEV-2013-0295 and SEV-20150496), CONSOLIDER Excellence Network (MAT2015-68994-REDC), COACHSUPENERGY project (MAT2014-51778-C2-1-R, co-financed by the European Regional Development Fund), and the projects MAT2011-28874-C02-01, ENE2014-56109-C3-3-R and Consolider Nanoselect CSD2007-00041, and from the Catalan Government with 2014-SGR-753, 2014-SGR-1638 and Xarmae. AQ and MdlM are also grateful to CSIC and European Social Fund program for JAE-Predoc fellowships (E-08-2012-1321248 and E-08-2013-1028356).

\section{REFERENCES}

(1) Barth, J. V.; Costantini, G.; Kern, K., Engineering atomic and molecular nanostructures at surfaces. Nature 2005, 437, 671-679.

(2) Yu, X.; Prevot, M. S.; Guijarro, N.; Sivula, K., Self-assembled 2D WSe 2 thin films for photoelectrochemical hydrogen production. Nat. Commun. 2015, 6, 7596.

(3) Arico, A. S.; Bruce, P.; Scrosati, B.; Tarascon, J.-M.; van Schalkwijk, W., Nanostructured materials for advanced energy conversion and storage devices. Nat. Mater. 2005, 4, 366-377.

(4) Teichert, C., Self-organization of nanostructures in semiconductor heteroepitaxy. Phys. Rep. 2002, $365,335-432$.

(5) Sun, S.; Murray, C. B.; Weller, D.; Folks, L.; Moser, A., Monodisperse FePt Nanoparticles and Ferromagnetic FePt Nanocrystal Superlattices. Science 2000, 287, 1989-1992.

(6) Brust, M.; Bethell, D.; Kiely, C. J.; Schiffrin, D. J., Self-Assembled Gold Nanoparticle Thin Films with Nonmetallic Optical and Electronic Properties. Langmuir 1998, 14, 5425-5429.

(7) Lopes, W. A.; Jaeger, H. M., Hierarchical self-assembly of metal nanostructures on diblock copolymer scaffolds. Nature 2001, 414, 735-738.

(8) Shchukin, V. A.; Bimberg, D., Spontaneous ordering of nanostructures on crystal surfaces. Rev. Mod. Phys. 1999, 71, 1125-1171.

(9) Brunner, K., Si/Ge nanostructures. Rep. Prog. Phys. 2002, 65, $27-72$.

(10) Schwartz, R. W.; Schneller, T.; Waser, R., Chemical solution deposition of electronic oxide films. C. R. Chim. 2004, 7, 433-461. 
(11) Vrejoiu, I.; Alexe, M.; Hesse, D.; Gosele, U., Functional Perovskites - From Epitaxial Films to Nanostructured Arrays. Adv. Funct. Mater. 2008, 18, 3892-3906.

(12) Dawber, M.; Rabe, K. M.; Scott, J. F., Physics of thin-film ferroelectric oxides. Rev. Mod. Phys. 2005, 77, 1083-1130.

(13) Tokura, Y., Critical features of colossal magnetoresistive manganites. Rep. Prog. Phys. 2006, 69, 797-851.

(14) Tokura, Y.; Hwang, H. Y., Condensed-matter physics: Complex oxides on fire. Nat. Mater. 2008, 7, 694-695.

(15) Ishii, Y.; Yamada, H.; Sato, H.; Akoh, H.; Ogawa, Y.; Kawasaki, M.; Tokura, Y., Improved tunneling magnetoresistance in interface engineered ( $\mathrm{La}, \mathrm{Sr}) \mathrm{MnO}_{3}$ junctions. Appl. Phys. Lett. 2006, 89, 042509.

(16) Llordes, A.; Palau, A.; Gazquez, J.; Coll, M.; Vlad, R.; Pomar, A.; Arbiol, J.; Guzman, R.; Ye, S.; Rouco, V.; Sandiumenge, F.; Ricart, S.; Puig, T.; Varela, M.; Chateigner, D.; Vanacken, J.; Gutierrez, J.; Moshchalkov, V.; Deutscher, G.; Magen, C.; Obradors, X., Nanoscale strain-induced pair suppression as a vortex-pinning mechanism in high-temperature superconductors. Nat. Mater. 2012, 11, 329-336.

(17) Bogle, K. A.; Cheung, J.; Chen, Y. L.; Liao, S. C.; Lai, C. H.; Chu, Y. H.; Gregg, J. M.; Ogale, S. B.; Valanoor, N., Epitaxial Magnetic Oxide Nanocrystals Via Phase Decomposition of Bismuth Perovskite Precursors. Adv. Funct. Mater. 2012, 22, 5224-5230.

(18) Cen, C.; Thiel, S.; Mannhart, J.; Levy, J., Oxide Nanoelectronics on Demand. Science 2009, 323, 1026-1030.

(19) Eerenstein, W.; Mathur, N. D.; Scott, J. F., Multiferroic and magnetoelectric materials. Nature 2006, 442, 759-765.

(20) Zhang, J.; Zhao, Y.; Zhao, X.; Liu, Z.; Chen, W., Porous Perovskite $\mathrm{LaNiO}_{3} \mathrm{Nanocubes}$ Cathode Catalysts for Li-O2 Batteries with Low Charge Potential. Sci. Rep. 2014, 4, 6005.

(21) Xu, J.-J.; Xu, D.; Wang, Z.-L.; Wang, H.-G.; Zhang, L.-L.; Zhang, X.-B., Synthesis of PerovskiteBased Porous $\mathrm{La}_{0.75} \mathrm{Sr}_{0.25} \mathrm{MnO}_{3}$ Nanotubes as a Highly Efficient Electrocatalyst for Rechargeable Lithium-Oxygen Batteries. Angew. Chem. Int. Ed. 2013, 52, 3887-3890.

(22) Fabbri, E.; Mohamed, R.; Levecque, P.; Conrad, O.; Kötz, R.; Schmidt, T. J., Composite Electrode Boosts the Activity of $\mathrm{Ba}_{0.5} \mathrm{Sr}_{0.5} \mathrm{Co}_{0.8} \mathrm{Fe}_{0.2} \mathrm{O}_{3-\delta}$ Perovskite and Carbon toward Oxygen Reduction in Alkaline Media. ACS Catal. 2014, 4, 1061-1070.

(23) Grinberg, I.; West, D. V.; Torres, M.; Gou, G.; Stein, D. M.; Wu, L.; Chen, G.; Gallo, E. M.; Akbashev, A. R.; Davies, P. K.; Spanier, J. E.; Rappe, A. M., Perovskite oxides for visible-light-absorbing ferroelectric and photovoltaic materials. Nature 2013, 503, 509-512.

(24) Hildebrandt, N. C.; Soldat, J.; Marschall, R., Layered Perovskite Nanofibers via Electrospinning for Overall Water Splitting. Small 2015, 11, 2051-2057.

(25) Sainz, M. A.; Durán, A.; Fernández Navarro, J. M., UV highly absorbent coatings with $\mathrm{CeO}_{2}$ and $\mathrm{TiO}_{2}$. J. Non-Cryst. Solids 1990, 121, 315-318.

(26) Krishna, M. G.; Hartridge, A.; Bhattacharya, A. K., Temperature and ionic size dependence of the properties of ceria based optionic thin films. Mat. Sci. Eng. B 1998, 55, 14-20.

(27) Porqueras, I.; Person, C.; Corbella, C.; Vives, M.; Pinyol, A.; Bertran, E., Characteristics of ebeam deposited electrochromic $\mathrm{CeO}_{2}$ thin films. Solid State Ion. 2003, 165, 131-137.

(28) Copetti, C. A.; Soltner, H.; Schubert, J.; Zander, W.; Hollricher, O.; Buchal, C.; Schulz, H.; Tellmann, N.; Klein, N., High quality epitaxy of $\mathrm{YBa}_{2} \mathrm{Cu}_{3} \mathrm{O}_{7-x}$ on silicon-on-sapphire with the multiple buffer layer YSZ/CeO 2 . Appl. Phys. Lett. 1993, 63, 1429-1431.

(29) Tye, L.; El-Masry, N. A.; Chikyow, T.; McLarty, P.; Bedair, S. M., Electrical characteristics of epitaxial $\mathrm{CeO}_{2}$ on $\mathrm{Si}(111)$. Appl. Phys. Lett. 1994, 65, 3081-3083.

(30) Obradors, X.; Puig, T.; Pomar, A.; Sandiumenge, F.; Mestres, N.; Coll, M.; Cavallaro, A.; Roma, N.; Gazquez, J.; Gonzalez, J. C.; Castano, O.; Gutierrez, J.; Palau, A.; Zalamova, K.; Morlens, S.; Hassini, A.; Gibert, M.; Ricart, S.; Moreto, J. M.; Pinol, S.; Isfort, D.; Bock, J., Progress towards all-chemical superconducting $\mathrm{YBa}_{2} \mathrm{Cu}_{3} \mathrm{O}_{7}$-coated conductors. Supercond. Sci. Technol. 2006, 19, S13-S26. 
(31) Feng, Z. A.; El Gabaly, F.; Ye, X.; Shen, Z.-X.; Chueh, W. C., Fast vacancy-mediated oxygen ion incorporation across the ceria-gas electrochemical interface. Nat. Commun. 2014, 5, 4374.

(32) Chueh, W. C.; Falter, C.; Abbott, M.; Scipio, D.; Furler, P.; Haile, S. M.; Steinfeld, A., High-Flux Solar-Driven Thermochemical Dissociation of $\mathrm{CO}_{2}$ and $\mathrm{H}_{2} \mathrm{O}$ Using Nonstoichiometric Ceria. Science 2010, 330, 1797-1801.

(33) Murray, E. P.; Tsai, T.; Barnett, S. A., A direct-methane fuel cell with a ceria-based anode. Nature 1999, 400, 649-651.

(34) Regoutz, A.; Egdell, R. G.; Wermeille, D.; Cowley, R. A.; Zhang, K. H. L., Strain and tilt during epitaxial growth of highly ordered $\mathrm{In}_{2} \mathrm{O}_{3}$ nanorods. Nanoscale 2013, 5, 7445-7451.

(35) Carretero-Genevrier, A.; Puig, T.; Obradors, X.; Mestres, N., Ferromagnetic 1D oxide nanostructures grown from chemical solutions in confined geometries. Chem. Soc. Rev. 2014, 43, 20422054.

(36) Sun, Y.; Liu, Q.; Gao, S.; Cheng, H.; Lei, F.; Sun, Z.; Jiang, Y.; Su, H.; Wei, S.; Xie, Y., Pits confined in ultrathin cerium(IV) oxide for studying catalytic centers in carbon monoxide oxidation. Nat. Commun. 2013, 4, 2899.

(37) Branda, M. M.; Ferullo, R. M.; Causà, M.; Illas, F., Relative Stabilities of Low Index and Stepped $\mathrm{CeO}_{2}$ Surfaces from Hybrid and GGA + U Implementations of Density Functional Theory. J. Phys. Chem. C 2011, 115, 3716-3721.

(38) Obradors, X.; Martínez-Julián, F.; Zalamova, K.; Vlad, V. R.; Pomar, A.; Palau, A.; Llordés, A.; Chen, H.; Coll, M.; Ricart, S.; Mestres, N.; Granados, X.; Puig, T.; Rikel, M., Nucleation and mesostrain influence on percolating critical currents of solution derived $\mathrm{YBa}_{2} \mathrm{Cu}_{3} \mathrm{O}_{7}$ superconducting thin films. Physica C Supercond. 2012, 482, 58-67.

(39) Meixner, M.; Schöll, E.; Shchukin, V. A.; Bimberg, D., Self-Assembled Quantum Dots: Crossover from Kinetically Controlled to Thermodynamically Limited Growth. Phys. Rev. Lett. 2001, 87, 236101.

(40) Rastelli, A.; Stoffel, M.; Tersoff, J.; Kar, G. S.; Schmidt, O. G., Kinetic evolution and equilibrium morphology of strained islands. Phys. Rev. Lett. 2005, 95, 026103.

(41) Goldfarb, I.; Banks-Sills, L.; Eliasi, R., Is the Elongation of Ge Huts in the Low-Temperature Regime Governed by Kinetics? Phys. Rev. Lett. 2006, 97, 206101.

(42) Aqua, J. N.; Berbezier, I.; Favre, L.; Frisch, T.; Ronda, A., Growth and self-organization of SiGe nanostructures. Phys. Rep. 2013, 522, 59-189.

(43) Dubrovskii, V. G., Nucleation Theory and Growth of Nanostructures. ed.; Springer: Berlin, 2014.

(44) Porter, D. A.; Easterling, K. E., Phase Transformations in Metals and Alloys. ed.; Chapman \& Hall: London, 1992.

(45) Lee, S.; Daruka, I.; Kim, C. S.; Barabási, A. L.; Merz, J. L.; Furdyna, J. K., Dynamics of Ripening of Self-Assembled II-VI Semiconductor Quantum Dots. Phys. Rev. Lett. 1998, 81, 3479-3482.

(46) Tersoff, J.; Tromp, R. M., Shape Transition in Growth of Strained Islands - Spontaneous Formation of Quantum Wires. Phys. Rev. Lett. 1993, 70, 2782-2785.

(47) Li, A.; Liu, F.; Petrovykh, D. Y.; Lin, J. L.; Viernow, J.; Himpsel, F. J.; Lagally, M. G., Creation of "Quantum Platelets" via Strain-Controlled Self-Organization at Steps. Phys. Rev. Lett. 2000, 85, 53805383.

(48) Obradors, X.; Puig, T.; Gibert, M.; Queralto, A.; Zabaleta, J.; Mestres, N., Chemical solution route to self-assembled epitaxial oxide nanostructures. Chem. Soc. Rev. 2014, 43, 2200-2225.

(49) Schneller, T.; Waser, R.; Kosec, M.; Payne, D., Chemical Solution Deposition of Functional Oxide Thin Films. ed.; Springer: London, 2013.

(50) Hasenkox, U.; Mitze, C.; Waser, R.; Arons, R. R.; Pommer, J.; Güntherodt, G., Chemical Solution Deposition of Epitaxial $\mathrm{La}_{1-\mathrm{x}}(\mathrm{Ca}, \mathrm{Sr})_{\mathrm{x}} \mathrm{MnO}_{3}$ Thin Films. J. Electroceram. 1999, 3, 255-260.

(51) Hayashi, M.; Yasui, S.; Funakubo, H.; Uchida, H., Preferential Crystal Growth of (100)-Oriented $\mathrm{BiFeO}_{3}$ Films on Si Substrate. IOP Conference Series: Materials Science and Engineering 2011, 18, 092033.

(52) Bassiri-Gharb, N.; Bastani, Y.; Bernal, A., Chemical solution growth of ferroelectric oxide thin films and nanostructures. Chem. Soc. Rev. 2014, 43, 2125-2140. 
(53) Zabaleta, J.; Jaafar, M.; Abellan, P.; Monton, C.; Iglesias-Freire, O.; Sandiumenge, F.; Ramos, C. A.; Zysler, R. D.; Puig, T.; Asenjo, A.; Mestres, N.; Obradors, X., Nanoscale magnetic structure and properties of solution-derived self-assembled $\mathrm{La}_{0.7} \mathrm{Sr}_{0.3} \mathrm{MnO}_{3}$ islands. J. Appl. Phys. 2012, 111, 024307.

(54) Queraltó, A.; de la Mata, M.; Arbiol, J.; Obradors, X.; Puig, T., Disentangling Epitaxial Growth Mechanisms of Solution Derived Functional Oxide Thin Films. Advanced Materials Interfaces 2016, 3 , 1600392.

(55) Queraltó, A.; Perez del Pino, A.; de la Mata, M.; Arbiol, J.; Tristany, M.; Obradors, X.; Puig, T., Ultrafast Epitaxial Growth of Functional Oxide Thin Films by Pulsed Laser Annealing of Chemical Solutions. Chem. Mater. 2016, 28, 6136-6145.

(56) Rupp, J. L. M.; Infortuna, A.; Gauckler, L. J., Microstrain and self-limited grain growth in nanocrystalline ceria ceramics. Acta Mater. 2006, 54, 1721-1730.

(57) Gibert, M.; Abellán, P.; Benedetti, A.; Puig, T.; Sandiumenge, F.; García, A.; Obradors, X., SelfOrganized $\mathrm{Ce}_{1-\mathrm{x}} \mathrm{Gd}_{\mathrm{x}} \mathrm{O}_{2-\mathrm{y}}$ Nanowire Networks with Very Fast Coarsening Driven by Attractive Elastic Interactions. Small 2010, 6, 2716-2724.

(58) Gibert, M.; Abellan, P.; Martinez, L.; Roman, E.; Crespi, A.; Sandiumenge, F.; Puig, T.; Obradors, $\mathrm{X}$., Orientation and shape selection of self-assembled epitaxial $\mathrm{Ce}_{1-\mathrm{x}} \mathrm{Gd}_{\mathrm{x}} \mathrm{O}_{2-\mathrm{y}}$ nanostructures grown by chemical solution deposition. CrystEngComm 2011, 13, 6719-6727.

(59) Gibert, M.; Garcia, A.; Puig, T.; Obradors, X., Thermodynamic stability analysis of isometric and elongated epitaxial $\mathrm{Ce}_{1-\mathrm{x}} \mathrm{Gd}_{\mathrm{x}} \mathrm{O}_{2-\mathrm{y}}$ nanostructures on perovskite substrates. Phys. Rev. B 2010, 82, 165415.

(60) Suzuki, H.; Miwa, Y.; Naoe, T.; Miyazaki, H.; Ota, T.; Fuji, M.; Takahashi, M., Orientation control and electrical properties of PZT/LNO capacitor through chemical solution deposition. J. Eur. Ceram. Soc. 2006, 26, 1953-1956.

(61) Chen, S.-T.; Wang, G.-S.; Zhang, Y.-Y.; Yang, L.-H.; Dong, X.-L., Orientation Control Growth of Lanthanum Nickelate Thin Films Using Chemical Solution Deposition. J. Am. Ceram. Soc. 2007, 90, 3635-3637.

(62) Griswold, E. M.; Weaver, L.; Sayer, M.; Calder, I. D., Phase transformations in rapid thermal processed lead zirconate titanate. J. Mater. Res. 1995, 10, 3149-3159.

(63) Queraltó, A.; de la Mata, M.; Martínez, L.; Magén, C.; Gibert, M.; Arbiol, J.; Hühne, R.; Obradors, $\mathrm{X}$; Puig, T., Orientation symmetry breaking in self-assembled $\mathrm{Ce}_{1-\mathrm{x}} \mathrm{Gd}_{\mathrm{x}} \mathrm{O}_{2-\mathrm{y}}$ nanowires derived from chemical solutions. RSC Adv. 2016, 6, 97226-97236.

(64) Shi, Y.; Bork, A. H.; Schweiger, S.; Rupp, J. L., The effect of mechanical twisting on oxygen ionic transport in solid-state energy conversion membranes. Nat. Mater. 2015, 14, 721-7.

(65) An, J.; Kim, Y. B.; Park, J.; Gur, T. M.; Prinz, F. B., Three-dimensional nanostructured bilayer solid oxide fuel cell with $1.3 \mathrm{~W} / \mathrm{cm}^{2}$ at 450 degrees C. Nano Lett. 2013, 13, 4551-5.

(66) Almar, L.; Tarancón, A.; Andreu, T.; Torrell, M.; Hu, Y.; Dezanneau, G.; Morata, A., Mesoporous ceramic oxides as humidity sensors: A case study for gadolinium-doped ceria. Sensor. Actuat. B-Chem. 2015, 216, 41-48.

(67) Kuhn, M.; Bishop, S. R.; Rupp, J. L. M.; Tuller, H. L., Structural characterization and oxygen nonstoichiometry of ceria-zirconia $\left(\mathrm{Ce}_{1-\mathrm{x}} \mathrm{Zr}_{\mathrm{x}} \mathrm{O}_{2-\delta}\right)$ solid solutions. Acta Mater. 2013, 61, (11), 4277-4288.

(68) Beie, H. J.; Gnörich, A., Oxygen gas sensors based on $\mathrm{CeO}_{2}$ thick and thin films. Sensor. Actuat. B-Chem. 1991, 4, 393-399.

(69) Jasinski, P.; Suzuki, T.; Anderson, H. U., Nanocrystalline undoped ceria oxygen sensor. Sensor. Actuat. B-Chem. 2003, 95, 73-77.

(70) Lu, H.-F.; Zhou, Y.; Han, W.-F.; Huang, H.-F.; Chen, Y.-F., High thermal stability of ceria-based mixed oxide catalysts supported on $\mathrm{ZrO}_{2}$ for toluene combustion. Catal. Sci. Tech. 2013, 3, 1480-1484.

(71) Maciel, C. G.; Silva, T. d. F.; Profeti, L. P. R.; Assaf, E. M.; Assaf, J. M., Study of CuO/CeO 2 catalyst with for preferential $\mathrm{CO}$ oxidation reaction in hydrogen-rich feed (PROX-CO). Appl. Catal. AGen 2012, 431-432, 25-32.

(72) Divins, N. J.; Angurell, I.; Escudero, C.; Pérez-Dieste, V.; Llorca, J., Influence of the support on surface rearrangements of bimetallic nanoparticles in real catalysts. Science 2014, 346, 620-623. 
(73) Mckay, M. R.; Shumway, J.; Drucker, J., Real-time coarsening dynamics of GeSi(100) nanostructures. J. Appl. Phys. 2006, 99, 094305.

(74) Rodriguez, J. A.; Graciani, J.; Evans, J.; Park, J. B.; Yang, F.; Stacchiola, D.; Senanayake, S. D.; Ma, S.; Pérez, M.; Liu, P.; Sanz, J. F.; Hrbek, J., Water-Gas Shift Reaction on a Highly Active Inverse $\mathrm{CeO}_{x} / \mathrm{Cu}(111)$ Catalyst: Unique Role of Ceria Nanoparticles. Angew. Chem. 2009, 121, 8191-8194.

(75) Fronzi, M.; Piccinin, S.; Delley, B.; Traversa, E.; Stampfl, C., Water adsorption on the stoichiometric and reduced $\mathrm{CeO}_{2}(111)$ surface: a first-principles investigation. Phys. Chem. Chem. Phys. 2009, 11, 9188-9199.

(76) Barth, C.; Laffon, C.; Olbrich, R.; Ranguis, A.; Parent, P.; Reichling, M., A perfectly stoichiometric and flat $\mathrm{CeO}_{2}(111)$ surface on a bulk-like ceria film. Sci. Rep. 2016, 6, 21165.

(77) Graciani, J.; Mudiyanselage, K.; Xu, F.; Baber, A. E.; Evans, J.; Senanayake, S. D.; Stacchiola, D. J.; Liu, P.; Hrbek, J.; Sanz, J. F.; Rodriguez, J. A., Highly active copper-ceria and copper-ceria-titania catalysts for methanol synthesis from $\mathrm{CO}_{2}$. Science 2014, 345, 546-550.

(78) Senanayake, S. D.; Stacchiola, D.; Evans, J.; Estrella, M.; Barrio, L.; Pérez, M.; Hrbek, J.; Rodriguez, J. A., Probing the reaction intermediates for the water-gas shift over inverse $\mathrm{CeO}_{\mathrm{x}} / \mathrm{Au}\left(\begin{array}{lll}1 & 1 & 1\end{array}\right)$ catalysts. J. Catal. 2010, 271, 392-400.

(79) Roura, P.; Farjas, J.; Ricart, S.; Aklalouch, M.; Guzman, R.; Arbiol, J.; Puig, T.; Calleja, A.; PeñaRodríguez, O.; Garriga, M.; Obradors, X., Synthesis of nanocrystalline ceria thin films by lowtemperature thermal decomposition of Ce-propionate. Thin Solid Films 2012, 520, 1949-1953.

(80) Queraltó, A. Growth of functional oxide heterostructures from chemical solutions using advanced processing methodologies. Universitat Autònoma de Barcelona, Barcelona, 2015.

(81) Ballabio, G.; Bernasconi, M.; Pietrucci, F.; Serra, S., Ab initio study of yttria-stabilized cubic zirconia surfaces. Phys. Rev. B 2004, 70, 075417.

(82) Stengel, M., Electrostatic stability of insulating surfaces: Theory and applications. Phys. Rev. B 2011, 84, 205432.

(83) Nie, J. C.; Yamasaki, H.; Mawatari, Y., Self-assembled growth of $\mathrm{CeO}_{2}$ nanostructures on sapphire. Phys. Rev. B 2004, 70, 195421.

(84) Ansari, H. M.; Dixit, V.; Zimmerman, L. B.; Rauscher, M. D.; Dregia, S. A.; Akbar, S. A., Self Assembly of Nanoislands on YSZ-(001) Surface: A Mechanistic Approach Toward a Robust Process. Nano Lett. 2013, 13, 2116-2121.

(85) Ushakov, S. V.; Navrotsky, A., Direct measurements of fusion and phase transition enthalpies in lanthanum oxide. J. Mater. Res. 2011, 26, 845-847.

(86) Lyzenga, G. A.; Ahrens, T. J.; Mitchell, A. C., Shock temperatures of $\mathrm{SiO}_{2}$ and their geophysical implications. J. Geophys. Res. Sol. Ea. 1983, 88, 2431-2444.

(87) Perry, D. L.; Phillips, S. L., Handbook of Inorganic Compounds. 2nd ed.; CRC Press: New York, 2011.

(88) Ostwald, W., Studies on the formation and transformation of solid bodies. Z. Phys. Chem. 1897, 22, 289-330.

(89) Ratke, L.; Voorhees, P. W., Growth and Coarsening: Ostwald Ripening in Material Processing. ed.; Springer: Berlin, 2002.

(90) Beysens, D.; Knobler, C. M.; Schaffar, H., Scaling in the growth of aggregates on a surface. Phys. Rev. B 1990, 41, 9814-9818.

(91) Bardotti, L.; Bartelt, M. C.; Jenks, C. J.; Stoldt, C. R.; Wen, J. M.; Zhang, C. M.; Thiel, P. A.; Evans, J. W., Formation and Equilibration of Submonolayer Island Distributions in $\mathrm{Ag} / \mathrm{Ag}(100)$ Homoepitaxy. Langmuir 1998, 14, 1487-1492.

(92) Yang, W. C.; Zeman, M.; Ade, H.; Nemanich, R. J., Attractive Migration and Coalescence: A Significant Process in the Coarsening of $\mathrm{TiSi}_{2}$ Islands on the Si(111) Surface. Phys. Rev. Lett. 2003, 90, 136102.

(93) Burke, J. E.; Turnbull, D., Recrystallization and grain growth. Prog. Metal Phys. 1952, 3, 220292. 
(94) Mehrer, H., Diffusion in Solids: Fundamentals, Methods, Materials, Diffusion-Controlled Processes. ed.; Springer: Berlin, 2007; Vol. 155.

(95) Löffler, J. F.; Johnson, W. L., Model for decomposition and nanocrystallization of deeply undercooled $\mathrm{Zr}_{41.2} \mathrm{Ti}_{13.8} \mathrm{Cu}_{12.5} \mathrm{Ni}_{10} \mathrm{Be}_{22.5}$. Appl. Phys. Lett. 2000, 76, 3394-3396.

(96) Rupp, J. L. M.; Scherrer, B.; Harvey, A. S.; Gauckler, L. J., Crystallization and Grain Growth Kinetics for Precipitation-Based Ceramics: A Case Study on Amorphous Ceria Thin Films from Spray Pyrolysis. Adv. Funct. Mater. 2009, 19, 2790-2799.

(97) Rupp, J. L. M.; Scherrer, B.; Gauckler, L. J., Engineering disorder in precipitation-based nanoscaled metal oxide thin films. Phys. Chem. Chem. Phys. 2010, 12, 11114-11124.

(98) Heiroth, S.; Frison, R.; Rupp, J. L. M.; Lippert, T.; Barthazy Meier, E. J.; Müller Gubler, E.; Döbeli, M.; Conder, K.; Wokaun, A.; Gauckler, L. J., Crystallization and grain growth characteristics of yttria-stabilized zirconia thin films grown by pulsed laser deposition. Solid State Ion. 2011, 191, 12-23.

(99) Jesson, D. E.; Chen, G.; Chen, K. M.; Pennycook, S. J., Self-Limiting Growth of Strained Faceted Islands. Phys. Rev. Lett. 1998, 80, 5156-5159.

(100) Kuo, C.-W.; Lee, Y.-H.; Hung, I. M.; Wang, M.-C.; Wen, S.-B.; Fung, K.-Z.; Shih, C.-J., Crystallization kinetics and growth mechanism of $8 \mathrm{~mol} \%$ yttria-stabilized zirconia (8YSZ) nano-powders prepared by a sol-gel process. J. Alloy. Compd. 2008, 453, 470-475.

(101) Ramanathan, S.; Muraleedharan, R. V.; Roy, S. K.; K, P.; Nayar, K., Dehydration and Crystallization Kinetics of Zirconia-Yttria Gels. J. Am. Ceram. Soc. 1995, 78, 429-432.

(102) Dubrovskii, V. G.; Xu, T.; Lambert, Y.; Nys, J. P.; Grandidier, B.; Stiévenard, D.; Chen, W.; Pareige, P., Narrowing the Length Distribution of Ge Nanowires. Phys. Rev. Lett. 2012, 108, 105501.

(103) Barabási, A.-L., Self-assembled island formation in heteroepitaxial growth. Appl. Phys. Lett. 1997, 70, 2565-2567. 